\title{
COHESIVE ELASTICITY AND SURFACE PHENOMENA
}

\author{
BY \\ CHIEN H. WU \\ University of Illinois at Chicago, Chicago, Illinois
}

\begin{abstract}
Cohesive elasticity is the grade-3 theory of elasticity developed by Mindlin in 1965. It has a modulus of cohesion that gives rise to surface-tension. The concept of adhesion is introduced, and interfacial energies and energy of adhesion are defined. The interfacial energy solution may also be used to define a grain boundary energy. Also presented are the thin film energy and the concept of an interface-phase. The stretching of a thin film is analyzed in detail; and it is found that the apparent Young's modulus obtained from a film is higher than that obtained from a plate.
\end{abstract}

1. Introduction. Every continuum field theory contains only a limited amount of physics, and even the limited amount of physics is very often only of a phenomenological nature in the sense that the various constants employed in the theory are closely related to but not directly derived from more fundamental physical parameters. It could be argued that Young's modulus should be directly derived from more fundamental bonding forces, but the "correct" Young's modulus has always been directly measured from a tension test. It is hard to define the exact physics content of the theory of elasticity, but it is perhaps reasonable to say that most of the socalled micromechanics phenomena we are so eager to understand are not derivable from the limited amount of physics that was built in elasticity. When the implications of a continuum theory have been exhaustively revealed by the mathematical solutions to the associated field equations, additional mathematical manipulations become redundant even though new solutions will always be of usefulness.

In terms of a lattice theory, elasticity incorporates only the nearest neighbor interactions, and that is it. The theory does not have an intrinsic length scale and, as a result, a $30 \mathrm{~cm}$ slab behaves the same as a $40 \mu \mathrm{m}$ film, and there is no difference between a microcrack and a geological fault. An intrinsic length scale appears when the forces between particles are extended to include first, second, and $n$th neighbor interactions. Moreover, an initial, homogeneous, self-equilibrating stress will lead to surface tension (Toupin and Gazis [25], Gazis and Wallis [10]). The surface-tension solution is of a boundary-layer type, and the associated decay constant has been estimated from electron-diffraction data obtained by Germer, MacRae, and Hartman [11].

Received August 14, 1990.

Supported by AFOSR under Grant-89-0503.

(C)1992 Brown University 
The continuum version of the $n$th neighbor-interaction lattice theory is the socalled grade- $n$ theory. If the strain energy-density is assumed to depend on the rotation-gradient, in addition to the strain, there results the couple stress theory. A complete grade- 2 theory depends on all eighteen components of the strain-gradient, and the additional stress quantity is sometimes termed the double stress (Toupin [24]). The inclusion of yet a third gradient, which has thirty independent components, leads to a grade- 3 theory and the associated new stress quantity is sometimes termed the triple stress (Mindlin [17]). There is also a very general theory that includes strain-gradients of any order (Green and Rivlin [12]).

Couple stress and higher-order gradient theories were popular topics of research in the sixties, and Mindlin's work is most noteworthy in that his goal was specifically targeted at understanding the effect of microstructures on the failure of solids (Mindlin [15-19]; Mindlin and Eshel [20]). In particular, and to the best of our knowledge, his grade-3 theory (Mindlin [17]) is the only continuum theory that is fully developed and has the capability of defining surface phenomena via nontrivial displacement fields. The most important concept of this theory is a new constant called the modulus of cohesion, which is essentially an initial, homogeneous, selfequilibrating triple stress. It is for this most important constant that we have coined the name cohesive elasticity to stress the significance of the theory.

The availability of self-equilibrating states is fully exploited in this paper. In addition to surface free energy, which was considered by Mindlin, we have introduced the concept of an adhesive joint to define various interfacial energies and energy of adhesion. When suitably defined, an interfacial energy becomes the grain boundary energy. Also introduced are the thin film energy and the concept of an interfacephase. The stretching of a thin film is analyzed at the end. It is shown that the apparent Young's modulus obtained from a film is higher than that obtained from a slab.

The basic equations are first recapitulated in a dimensionless form. Stress function representations are then presented in terms of solutions to thirteen uncoupled second-order equations. A complete boundary-layer solution is obtained for a regular boundary, and several significant, outstanding problems are outlined at the end.

2. Notation. We consider vectors in three-dimensional Euclidean space. Such vectors will be denoted by lowercase boldface letters $\left(\mathbf{a}, \mathbf{b}, \mathbf{n}\right.$, etc.). Let $\mathbf{e}_{1}, \mathbf{e}_{2}, \mathbf{e}_{3}$ denote the unit vectors in the directions of the three coordinate axes $z_{1}, z_{2}, z_{3}$ of a rectangular right-handed cartesian system with origin 0 . The position vector of a point $P$ relative to 0 is denoted by $\mathbf{z}$.

In addition to scalar product $\mathbf{a} \cdot \mathbf{b}$ and vector product $\mathbf{a} \times \mathbf{b}$ of the two vectors $\mathbf{a}$ and $\mathbf{b}$, the dyadic product is written $\mathbf{a} \otimes \mathbf{b}$, which, in case of no confusion, is also written ab. A second-order cartesian tensor $\mathbf{T}$ can be expressed as a linear combination of unit dyads, so that it takes the form

$$
\mathbf{T}=T_{i j} \mathbf{e}_{i} \otimes \mathbf{e}_{j},
$$

where the summation convention is employed. As a rule, we shall use boldface capitals to denote cartesian tensors of second (and higher) order. Moreover, an 
overscript is used to denote the order. Thus, a cartesian tensor of order $n$ can be expressed in components as

$$
\stackrel{n}{\mathbf{T}}=\underbrace{T_{i j \cdots m}}_{n \text { indices }} \underbrace{\mathbf{e}_{i} \otimes \mathbf{e}_{j} \otimes \cdots \otimes \mathbf{e}_{m}}_{n \text { factors }} .
$$

The inner product $\stackrel{n}{\mathbf{A}} \cdot \stackrel{m}{\mathbf{B}}$ and outer product $\stackrel{n}{\mathbf{A}} \otimes \stackrel{m}{\mathbf{B}}$ are illustrated by

$$
\begin{aligned}
& \stackrel{2}{\mathbf{A}} \cdot \stackrel{2}{\mathbf{B}}=A_{i j} B_{j k} \mathbf{e}_{i} \otimes \mathbf{e}_{k}, \\
& \stackrel{2}{\mathbf{A}} \otimes \stackrel{2}{\mathbf{B}}=A_{i j} B_{k l} \mathbf{e}_{i} \otimes \mathbf{e}_{j} \otimes \mathbf{e}_{k} \otimes \mathbf{e}_{l} .
\end{aligned}
$$

In addition, the rule of contraction $\stackrel{n}{\mathbf{A}} * \stackrel{m}{\mathbf{B}}$ of tensors is illustrated by

$$
\begin{gathered}
\left(\mathbf{a}_{1} \mathbf{a}_{2}\right) *\left(\mathbf{b}_{1} \mathbf{b}_{2} \mathbf{b}_{3}\right)=\left(\mathbf{a}_{1} \cdot \mathbf{b}_{1}\right)\left(\mathbf{a}_{2} \cdot \mathbf{b}_{2}\right) \mathbf{b}_{3}, \\
\left(\mathbf{a}_{1} \mathbf{a}_{2} \mathbf{a}_{3}\right) *\left(\mathbf{b}_{1} \mathbf{b}_{2}\right)=\mathbf{a}_{1}\left(\mathbf{a}_{2} \cdot \mathbf{b}_{1}\right)\left(\mathbf{a}_{3} \cdot \mathbf{b}_{2}\right), \\
\left(\mathbf{a}_{1} \mathbf{a}_{2} \mathbf{a}_{3}\right) *\left(\mathbf{b}_{1} \mathbf{b}_{2} \mathbf{b}_{3}\right)=\left(\mathbf{a}_{1} \cdot \mathbf{b}_{1}\right)\left(\mathbf{a}_{2} \cdot \mathbf{b}_{2}\right)\left(\mathbf{a}_{3} \cdot \mathbf{b}_{3}\right),
\end{gathered}
$$

where, for example, $\left(\mathbf{a}_{1} \mathbf{a}_{2}\right)=\mathbf{a}_{1} \otimes \mathbf{a}_{2}$. For convenience, the dyadic product indicator $\otimes$ will not be explicitly shown in places where no apparent confusion will result from the omission.

The theory developed by Mindlin involves a material length-scale $l$, which is assumed to be much smaller than the geometric length-scale $L$ associated with a problem. The ratio $\varepsilon=l / L$ plays a key role in the theory, as well as in the ensuing asymptotic analyses and results. It is for this reason that we have chosen to recapitulate the theory in a dimensionless form.

Throughout this paper the length-scale $L$ is prefixed so that, e.g.,

$$
\operatorname{Dim} . \mathbf{z}=L \mathbf{z},
$$

and hence $z_{i}$ are dimensionless. Let $\mu$ be the shear modulus. The force-scale used throughout this paper is just $L^{2} \mu$. Also, the notation Dim.( ) is used to mean dimensional form of ( ), instead of dimension of ( ).

3. Governing equations. Let $\mathbf{u}(\mathbf{z})$ be the dimensionless displacement vector. Following the original work of Mindlin [17], the dimensionless strain energy-density, $W$, is assumed to be a function of three displacement-gradient tensors,

$$
W=W(\stackrel{2}{\mathbf{E}}, \stackrel{3}{\mathbf{E}}, \stackrel{4}{\mathbf{E}})
$$

where

$$
\begin{aligned}
& \stackrel{2}{\mathbf{E}}=\frac{1}{2}(\boldsymbol{\nabla} \mathbf{u}+\mathbf{u} \nabla), \quad E_{i j}=\frac{1}{2}\left(u_{i, j}+u_{j, i}\right), \\
& \stackrel{3}{\mathbf{E}}=\nabla \nabla \mathbf{u}, \quad E_{i j k}=u_{k, i j},
\end{aligned}
$$

\footnotetext{
${ }^{1}$ Multiply $W$ by $\mu$ to recover its dimension.
} 


$$
\stackrel{4}{\mathbf{E}}=\nabla \nabla \nabla \mathbf{u}, \quad E_{i j k l}=u_{l, i j k},
$$

and $\boldsymbol{\nabla}$ is the gradient operator in the dimensionless $\mathbf{z}$. The symmetry properties of the above are self-explanatory.

The variation of the total strain energy, in a (dimensionless) volume $V$, with arbitrary variation of $\mathbf{u}$, is

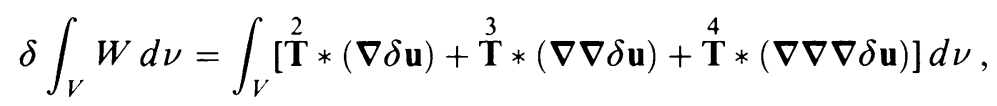

where $d \nu=d z_{1} d z_{2} d z_{3}$ and

$$
\stackrel{2}{\mathbf{T}}=\partial W / \partial \stackrel{2}{\mathbf{E}}, \quad \stackrel{3}{\mathbf{T}}=\partial W / \partial \stackrel{3}{\mathbf{E}}, \quad \stackrel{4}{\mathbf{T}}=\partial W / \partial \stackrel{4}{\mathbf{E}} .
$$

The right-hand side of (3.5) may be reduced to include a surface integral by application of the chain rule of differentiation and the divergence theorem. In doing so, however, additional caution must be taken to recognize the fact that $\nabla \delta \mathbf{u}$, together with other similar terms, is not independent of $\delta \mathbf{u}$ on the (dimensionless) surface $\partial V$ of the volume $V$.

A number of surface operations and surface operators are needed to complete the desired reduction. Let $\mathbf{n}$ be the unit normal to $\partial V$ and pointing away from $V$. The following are applicable and defined on $\partial V$ :

$$
\begin{gathered}
\boldsymbol{\nabla} \delta \mathbf{u}=\mathbf{n} \partial_{n} \delta \mathbf{u}+\nabla^{0} \delta \mathbf{u}, \\
\delta_{n} \equiv \mathbf{n} \cdot \boldsymbol{\nabla}, \\
\boldsymbol{\nabla}^{0} \equiv(\mathbf{I}-\mathbf{n n}) \cdot \boldsymbol{\nabla}, \\
\boldsymbol{\nabla}^{\prime} \equiv \mathbf{n}\left(\nabla^{0} \cdot \mathbf{n}\right)-\nabla^{0}=\left(\frac{1}{R_{1}}+\frac{1}{R_{2}}\right) \mathbf{n}-\nabla^{0},
\end{gathered}
$$

where $I$ is the unit tensor and $\left(1 / R_{1}+1 / R_{2}\right)$ the mean curvature of the surface. It is noted that for a flat surface, $\nabla^{\prime}$ is just the negative of $\nabla^{0}$, the plane gradient operator.

Using the above relations, from (3.5) we obtain

$$
\begin{aligned}
& \delta \int_{V} W d \nu=-\int_{V}\left\{\boldsymbol{\nabla} \cdot\left[\mathbf{T}^{2}-\boldsymbol{\nabla} \cdot \mathbf{T}^{3}+\boldsymbol{\nabla} \cdot(\boldsymbol{\nabla} \cdot \mathbf{4})\right]\right\} \cdot \delta \mathbf{u} d \nu
\end{aligned}
$$

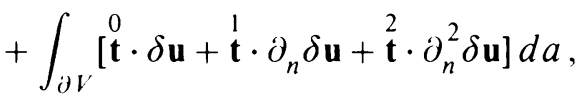

where

$$
\begin{aligned}
& { }^{0}=\mathbf{n} \cdot[\stackrel{2}{\mathbf{T}}-\boldsymbol{\nabla} \cdot \stackrel{3}{\mathbf{T}}+(\boldsymbol{\nabla} \boldsymbol{\nabla}) * \stackrel{4}{\mathbf{T}}]+\boldsymbol{\nabla}^{\prime} \cdot\left[\boldsymbol{\nabla}^{\prime} \cdot(\mathbf{n} \cdot \stackrel{4}{\mathbf{T}})\right] \\
& +\boldsymbol{\nabla}^{\prime} \cdot\left\{\mathbf{n} \cdot[\stackrel{3}{\mathbf{T}}-\boldsymbol{\nabla} \cdot \stackrel{4}{\mathbf{T}}]-\left(\boldsymbol{\nabla}^{0} \mathbf{n}\right) \cdot[(\mathbf{n n}) * \stackrel{4}{\mathbf{T}}]\right\}, \\
& { }^{\prime}=(\mathbf{n n}) *(\stackrel{3}{\mathbf{T}}-\boldsymbol{\nabla} \cdot \stackrel{4}{\mathbf{T}})+\mathbf{n} \cdot\left[\boldsymbol{\nabla}^{\prime} \cdot(n \cdot \stackrel{4}{\mathbf{T}})\right]+\boldsymbol{\nabla}^{\prime} \cdot[(\mathbf{n n}) * \stackrel{4}{\mathbf{T}}],
\end{aligned}
$$

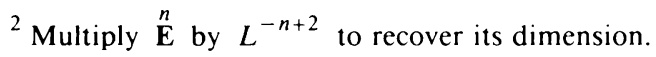

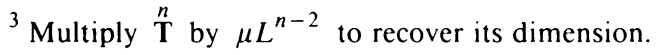




$$
\stackrel{2}{\mathbf{t}}=(\mathbf{n n n}) * \stackrel{4}{\mathbf{T}},
$$

are the generalized surface traction vectors associated with the generalized surface kinematic vectors $\mathbf{u}, \partial_{n} \mathbf{u}$, and $\partial_{n}^{2} \mathbf{u}$, respectively. The Generalized Principle of Stationary Potential Energy and stress-equation of equilibrium are

$$
\begin{aligned}
& \delta \int_{V} W d \nu=\int_{V} \mathbf{f} \cdot \delta \mathbf{u} d \nu+\int_{\partial V}\left[\stackrel{0}{\mathbf{t}} \cdot \delta \mathbf{u}+\stackrel{1}{\mathbf{t}} \cdot \partial_{n} \delta \mathbf{u}+\stackrel{2}{\mathbf{t}} \cdot \partial_{n}^{2} \delta \mathbf{u}\right] d a, \\
& \boldsymbol{\nabla} \cdot[\stackrel{2}{\mathbf{T}}-\boldsymbol{\nabla} \cdot \stackrel{3}{\mathbf{T}}+(\boldsymbol{\nabla} \nabla) * \stackrel{4}{\mathbf{T}}]+\mathbf{f}=0 .
\end{aligned}
$$

For homogeneous and isotropic materials, the following 2 nd degree polynomial $W$ was deduced by Mindlin:

$$
\begin{aligned}
W= & {\left[E_{i j} E_{i j}+\frac{3-\kappa}{2(\kappa-1)} E_{i i} E_{j j}\right]+\varepsilon^{2} \beta_{0} E_{i i j j} } \\
& +\varepsilon^{2}\left[\alpha_{1} E_{i j j} E_{i k k}+\alpha_{2} E_{i i k} E_{k j j}+\alpha_{3} E_{i i k} E_{j j k}+\alpha_{4} E_{i j k} E_{i j k}+\alpha_{5} E_{i j k} E_{k j i}\right] \\
& +\varepsilon^{4}\left[\beta_{1} E_{i i j j} E_{k k l l}+\beta_{2} E_{i j k k} E_{i j l l}+\beta_{3} E_{i i j k} E_{j k l l}\right. \\
& \left.\quad+\beta_{4} E_{i i j k} E_{l l k j}+\beta_{5} E_{i i j k} E_{l l j k}+\beta_{6} E_{i j k l} E_{i j k l}+\beta_{7} E_{i j k l} E_{j k l i}\right] \\
& +\varepsilon^{2}\left[\gamma_{1} E_{i i} E_{j j k k}+\gamma_{2} E_{i j} E_{i j k k}+\gamma_{3} E_{i j} E_{k k j j}\right]
\end{aligned}
$$

where $\varepsilon=l / L$,

$$
\kappa= \begin{cases}3-4 \nu & (\text { general and plane strain }) \\ \frac{3-\nu}{1+\nu} & (\text { plane stress of elasticity })\end{cases}
$$

and $\nu$ is Poisson's ratio. All the remaining constants are expressed in dimensionless form via $\mu$ and a material length-scale $l$, which is assumed to be small so that $\varepsilon \ll 1$. We shall refer to $\varepsilon$ as the small parameter from time to time. For example, as the small parameter tends to zero, (3.17) tends to the strain energy-density of the theory of linear elasticity. All important mathematical and physical implications of the assumed $W$ may be found in Mindlin's original work and are amplified by the explicit introduction of $l$ and $\varepsilon$. Finally, the material constants introduced by Mindlin are

$$
\left\{a_{n}, b_{0}, b_{n}, c_{n}\right\}=\mu\left\{l^{2} \alpha_{n}, l^{2} \beta_{0}, l^{4} \beta_{n}, l^{2} \gamma_{n}\right\} .
$$

From (3.6) and (3.17) follow the constitutive equations:

$$
\begin{aligned}
& T_{p q}=\left[2 E_{p q}+\frac{3-\kappa}{\kappa-1} E_{i i} \delta_{p q}\right]+\varepsilon^{2}\left[\gamma_{1} E_{i i j j} \delta_{p q}+\gamma_{2} E_{p q i i}+\frac{1}{2} \gamma_{3}\left(E_{i i p q}+E_{i i q p}\right)\right] \\
& T_{p q r}=\varepsilon^{2}\left[\alpha_{1}\left(E_{p i i} \delta_{q r}+E_{q i i} \delta_{p r}\right)\right.+\frac{1}{2} \alpha_{2}\left(E_{i i p} \delta_{q r}+2 E_{r i i} \delta_{q p}+E_{i i q} \delta_{p r}\right) \\
&\left.+2 \alpha_{3} E_{i i r} \delta_{p q}+2 \alpha_{4} E_{p q r}+\alpha_{5}\left(E_{r q p}+E_{r p q}\right)\right]
\end{aligned}
$$

${ }^{4}$ Multiply ${ }^{n}$ by $\mu L^{n}$ to recover its dimension.

${ }^{5}$ The dimensional forms of the various terms are illustrated by, e.g., $\varepsilon^{2} \beta_{0} E_{i i j j} \rightarrow\left(\mu l^{2} \beta_{0}\right)\left(E_{i i j j} / L_{2}\right)$.

${ }^{6}$ The meaning of plane stress in the new theory should be reexamined. 


$$
\begin{aligned}
T_{p q r s}= & \varepsilon^{4}\left\{\frac{2}{3} \beta_{1} E_{i i j j} \delta_{p q r s}+\frac{2}{3} \beta_{2} E_{j k i i} \delta_{j k p q r s} \frac{1}{6} \beta_{3}\left[\left(E_{i i j k}+E_{i i k j}\right) \delta_{j k p q r s}+2 E_{j s i i} \delta_{j p q r}\right]\right. \\
& \left.+\frac{2}{3} \beta_{4} E_{i i s j} \delta_{j p q r}+\frac{2}{3} \beta_{5} E_{i i j s} \delta_{j p q r}+2 \beta_{6} E_{p q r s}+\frac{2}{3} \beta_{7}\left(E_{q r s p}+E_{r s p q}+E_{s p q r}\right)\right\} \\
& +\varepsilon^{2}\left[\frac{1}{3} \gamma_{1} E_{i i} \delta_{p q r s}+\frac{1}{3} \gamma_{2} E_{i j} \delta_{i j p q r s}+\frac{1}{3} \gamma_{3} E_{i s} \delta_{i p q r}\right]+\varepsilon^{2} \frac{1}{3} \beta_{0} \delta_{p q r s},
\end{aligned}
$$

where $\delta_{i j}$ is the Kronecker delta and

$$
\begin{gathered}
\delta_{i j k l}=\delta_{i j} \delta_{k l}+\delta_{i k} \delta_{j l}+\delta_{i l} \delta_{j k}, \\
\delta_{i j k l m n}=\delta_{i k} \delta_{j l} \delta_{m n}+\delta_{i k} \delta_{j m} \delta_{l n}+\delta_{i l} \delta_{j m} \delta_{k n} .
\end{gathered}
$$

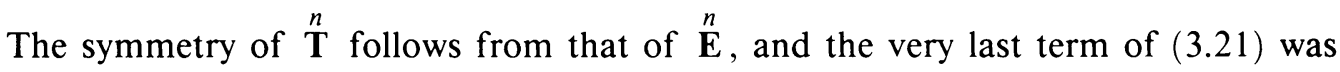
identified by Mindlin as a cohesive force that gives rise to surface tension. As we shall see, it also gives rise to interface-phase and interphase-interface energies.

Let $W^{*}[\mathbf{u}]$ be the total elastic energy. Then (3.17)-(3.21) lead to the conclusion that

$$
W^{*}=\int_{V} W d \nu=\frac{1}{2} \varepsilon^{2} \beta_{0} \int_{V} \nabla^{2} \nabla \cdot \mathbf{u} d \nu+\frac{1}{2} \int_{V}\left(\stackrel{2}{\mathbf{T}} * \stackrel{2}{\mathbf{E}}^{2}+\stackrel{3}{\mathbf{T}} * \stackrel{3}{\mathbf{E}}^{2} \stackrel{4}{\mathbf{T}} * \stackrel{4}{\mathbf{E}}\right) d \nu,
$$

where the appearance of the first integral is a result of the fact that the $\beta_{0}$-term in $W$ is linear in $E_{i i j j}$. The second volume integral may be converted into a surface integral by following the steps from (3.6)-(3.11), and the net outcome is the Generalized Clapeyron's Theorem

$$
2 \int_{V} W d \nu=\int_{V} \mathbf{f} \cdot \mathbf{u} d \nu+\varepsilon^{2} \beta_{0} \int_{V} \nabla^{2} \nabla \cdot \mathbf{u} d \nu+\int_{\partial V}\left[\begin{array}{l}
0 \\
\mathbf{t} \cdot \mathbf{u}
\end{array}+\mathbf{t} \cdot \partial_{n} \mathbf{u}+\stackrel{2}{\mathbf{t}} \cdot \partial_{n}^{2} \mathbf{u}\right] d a .
$$

Finally, the total potential energy $U^{*}$ of the system is

$$
U^{*}=W^{*}-\int_{V} \mathbf{f} \cdot \mathbf{u} d \nu-\int_{\partial V}\left[0, \mathbf{t} \cdot \mathbf{u}+\mathbf{t} \cdot \partial_{n} \mathbf{u}+\stackrel{2}{\mathbf{t}} \cdot \partial_{n}^{2} \mathbf{u}\right] d a .
$$

It follows from the above that if there are no extremal forces, i.e., if ${ }_{\mathbf{t}}^{0}, \mathbf{t}^{1}, 2$ $f$ are identically zero, then the total potential energy is merely

$$
U^{*}=W^{*}=\frac{1}{2} \varepsilon^{2} \beta_{0} \int_{V} \nabla^{2} \nabla \cdot \mathbf{u}^{(e)} d \nu=\frac{1}{2} \varepsilon^{2} \beta_{0} \int_{\partial V} \partial_{n} \nabla \cdot \mathbf{u}^{(e)} d a,
$$

where $\mathbf{u}=\mathbf{u}^{(e)}$, if it exists, is a self-equilibrating state. The total potential energy of the system, (3.26), is the free energy, either the Gibbs free energy or the Helmholtz free energy. When a traction-free surface is the source of the disturbance, the free energy is (3.27). It follows that the dimensionless surface free energy per unit surface area $F_{s}$ is given by

$$
F_{s}=\frac{1}{2} \varepsilon^{2} \beta_{0} \partial_{n} \nabla \cdot \mathbf{u}^{(e)},
$$

the dimensional form of which is termed the surface tension by Mindlin. We shall see that (3.26) can be used to define many surface related energies.

The displacement equation of equilibrium deduced from (3.16)-(3.21) is

$$
\frac{\kappa+1}{\kappa-1} D_{11}^{2} D_{12}^{2} \nabla \nabla \cdot \mathbf{u}-D_{21}^{2} D_{22}^{2} \boldsymbol{\nabla} \times \boldsymbol{\nabla} \times \mathbf{u}+\mathbf{f}=0,
$$


where

$$
D_{\lambda \mu}^{2} \equiv 1-\varepsilon^{2} \alpha_{\lambda \mu}^{2} \nabla^{2}, \quad \lambda=1,2 ; \mu=1,2
$$

and

$$
\begin{aligned}
2 \frac{\kappa+1}{\kappa-1} \alpha_{1 \lambda}^{2} & =\alpha-2 \gamma \pm\left[(\alpha-2 \gamma)^{2}-4 \beta \frac{\kappa+1}{\kappa-1}\right]^{1 / 2} \\
2 \alpha_{2 \lambda}^{2} & =\alpha^{\prime}-\gamma_{3} \pm\left[\left(\alpha^{\prime}-\gamma_{3}\right)^{2}-4 \beta^{\prime}\right]^{1 / 2}
\end{aligned}
$$

in which $\lambda=1,2$; and

$$
\begin{aligned}
& \alpha=2\left(\alpha_{1}+\alpha_{2}+\alpha_{3}+\alpha_{4}+\alpha_{5}\right), \quad \alpha^{\prime}=2\left(\alpha_{3}+\alpha_{4}\right), \\
& \beta=2\left(\beta_{1}+\beta_{2}+\beta_{3}+\beta_{4}+\beta_{5}+\beta_{6}+\beta_{7}\right), \quad \beta^{\prime}=2\left(\beta_{5}+\beta_{6}\right), \\
& \gamma=\gamma_{1}+\gamma_{2}+\gamma_{3} .
\end{aligned}
$$

As the small parameter $\varepsilon$ tends to zero, (3.29) reduces to the displacement equation of equilibrium of the theory of elasticity.

As it was pointed out by Mindlin that the conditions for positive $W$ do not include relations between $\alpha$ (or $\alpha^{\prime}$ ) and $\beta, \gamma$ (or $\beta^{\prime}, \gamma_{3}$ ) and hence, supply no indications of the character, real or complex, of the four constants $\alpha_{\lambda \mu}$. In all the specific solutions considered in the sequel, the $\alpha_{\lambda \mu}$ will be treated as if they were real and positive; but complex $\alpha_{\lambda \mu}$ are equally admissible. The character, real or complex, of the four constants $\alpha_{\lambda \mu}$ dictates the behavior of the field variables. On the other hand, for the most part, the final products of the problems to be analyzed are energy expressions of one form or another. These energies turn out to be independent of the character of $\alpha_{\lambda \mu}$. In any case the two pairs of constants $\left(\alpha_{11}, \alpha_{12}\right)$ and $\left(\alpha_{21}, \alpha_{22}\right)$ are assumed to be positive throughout this paper. In case they are actually complex, the following substitution is presumed:

$$
\begin{aligned}
& \alpha_{12}=\bar{\alpha}_{11}, \quad \operatorname{Real} \alpha_{11}>0 ; \\
& \alpha_{22}=\bar{\alpha}_{21}, \quad \operatorname{Real} \alpha_{21}>0 \text {; }
\end{aligned}
$$

which, of course, satisfy (3.31) and (3.32).

Equations (3.5), (3.11), together with the equilibrium equation (3.16), yield the identity

$$
\begin{aligned}
\int_{V}\left[\stackrel{2}{\mathbf{T}}^{*}(\nabla \delta \mathbf{u})+\stackrel{3}{\mathbf{T}}^{*}(\nabla \nabla \delta \mathbf{u})+\stackrel{4}{\mathbf{T}}(\nabla \nabla \nabla \delta \mathbf{u})\right] d \nu \\
\quad=\int_{V} \mathbf{f} \cdot \delta \mathbf{u} d \nu+\int_{\partial V}\left[\stackrel{0}{\mathbf{t}} \cdot \delta \mathbf{u}+\stackrel{1}{\mathbf{t}} \cdot \partial_{n} \delta \mathbf{u}+\stackrel{2}{\mathbf{t}} \cdot \partial_{n}^{2} \delta \mathbf{u}\right] d a,
\end{aligned}
$$

which may be used to establish certain useful integral identities. The result of setting $\delta \mathbf{u}=\mathbf{c}$, an arbitrary constant vector, is

$$
\mathbf{c} \cdot\left[\int_{V} \mathbf{f} d \nu+\int_{\partial V}^{\stackrel{0}{\mathbf{t}} d a}\right]=0,
$$

and the vanishing of the resultant force is a necessary condition for (3.16) to have a solution. The result of setting $\delta \mathbf{u}=\boldsymbol{\omega} \times \mathbf{z}$, where $\boldsymbol{\omega}$ is an arbitrary constant vector, is

$$
\boldsymbol{\omega} \cdot\left[\int_{V} \mathbf{z} \times \mathbf{f} d \nu+\int_{\partial V}\left(\mathbf{z} \times \mathbf{t}^{0}+\mathbf{n} \times \stackrel{1}{\mathbf{t}}\right) d a\right]=0,
$$


and, hence, the vanishing of the moment becomes another necessary condition. The rest of $\mathbf{t}$ and ${ }_{\mathbf{t}}^{2}$, i.e., $\mathbf{t} \cdot \mathbf{n}$ and the full ${ }_{\mathbf{t}}^{2}$, are self-equilibrating and, hence, may be arbitrarily specified.

It is possible to obtain additional integral identities from (3.36), which may become useful in establishing the expected new Eshelby $[6,7,9]$ tensors and the associated conservation laws (Knowles and Sternberg [14], Budiansky and Rice [1]), but the possibility is not pursued further in this paper.

The stress equation of equilibrium, (3.16), may be written as

$$
\boldsymbol{\nabla} \cdot \mathbf{T}^{c}+\mathbf{f}=0
$$

where

$$
\mathbf{T}^{c} \equiv \stackrel{2}{\mathbf{T}}-\nabla \cdot \stackrel{3}{\mathbf{T}}+\nabla \cdot(\boldsymbol{\nabla} \cdot \stackrel{4}{\mathbf{T}})
$$

may be considered as the Cauchy stress tensor with the associated Cauchy stress vector $\mathbf{t}^{c}$ defined by

$$
\mathbf{t}^{c}=\mathbf{n} \cdot \mathbf{T}^{c}=\stackrel{0}{\mathbf{t}}-\boldsymbol{\nabla}^{\prime} \cdot\{\cdots\},
$$

where $\nabla^{\prime} \cdot\{\cdots\}$ may be identified with the terms given in (3.12). It is clear that

$$
\begin{gathered}
\int_{V} \mathbf{f} d \nu+\int_{\partial V} \mathbf{t}^{c} d a=0, \\
\int_{V} \mathbf{z} \times \mathbf{f} d \nu+\int_{\partial V} \mathbf{z} \times \mathbf{t}^{c} d a=0 .
\end{gathered}
$$

The following surface integral identity will enable us to show that (3.37), (3.38) are equivalent to the above.

Let $\mathbf{A}$ be an arbitrary second-order tensor. We have

$$
\begin{aligned}
\int_{\partial V} \boldsymbol{\nabla}^{0} \cdot \mathbf{A} d a & =\int_{\partial V} \boldsymbol{\nabla}^{0} \cdot\{\mathbf{n}(\mathbf{n} \cdot \mathbf{A})+[\mathbf{A}-\mathbf{n}(\mathbf{n} \cdot \mathbf{A})]\} d a \\
& =\int_{\partial V}\left(\boldsymbol{\nabla}^{0} \cdot \mathbf{n}\right)(\mathbf{n} \cdot \mathbf{A}) d a+\int_{\partial V} \nabla^{0} \cdot[\mathbf{A}-\mathbf{n}(\mathbf{n} \cdot \mathbf{A})] d a,
\end{aligned}
$$

where $\nabla^{0}$ is the plane gradient operator defined by (3.9). For a smooth closed surface $\partial V$, the last surface integral vanishes. It follows that

$$
\int_{\partial V}\left[\left(\boldsymbol{\nabla}^{0} \cdot \mathbf{n}\right)(\mathbf{n} \cdot \mathbf{A})-\nabla^{0} \cdot \mathbf{A}\right] d a=\int_{\partial V} \boldsymbol{\nabla}^{\prime} \cdot \mathbf{A} d a=0,
$$

which is the desired identity.

In the usual continuum theory of thermodynamics, the work term is axiomatically defined to be

$$
\int_{\partial V} \mathbf{n} \cdot \mathbf{T}^{c} \cdot \dot{\mathbf{u}} d a
$$

even if $\mathbf{T}^{c}$ is assumed to depend on $\nabla \mathbf{u}, \nabla \nabla \mathbf{u}$, and $\nabla \nabla \nabla \mathbf{u}$. This led Dunn and Serrin [5] to conclude that "a troubling aspect of all higher-grade models is that they are in general incompatible with the usual continuum theory of thermodynamics". They then proceeded to introduce the so-called interstitial working into the work integral. Mindlin's derivation, however, clearly indicates that the correct work term 
is the surface integral of (3.26), and an appropriately modified continuum theory would lead to thermodynamic compatibility. Similar discrepancies also show up in other higher-order gradient theories (see, e.g., Gurtin [13]).

4. Stress functions. The solution to the displacement equation of equilibrium was obtained by Mindlin in terms of the solutions to four uncoupled partial differential equations of order six. We give a more direct deduction and show that Mindlin's representation can be further simplified. Moreover, our deduction is constructive in that it lends itself to the actual construction of general solutions. The well-known Galerkin-Somigliana and Papkovich-Neuber representations serve as the starting point, and the component-function approach employed by Doyle [4] is then used to reduce the system to a set of thirteen uncoupled 2 nd order equations.

The displacement equation of equilibrium (3.29) is first rewritten in the form

$$
\left[\left(\frac{\kappa+1}{\kappa-1} D_{11}^{2} D_{12}^{2}-D_{21}^{2} D_{22}^{2}\right) \nabla \nabla \cdot \mathbf{u}+D_{21}^{2} D_{22}^{2} \nabla^{2} \mathbf{u}\right]+\mathbf{f}=0 .
$$

In case of zero body force, the divergence of the above yields

$$
D_{11}^{2} D_{12}^{2} \nabla^{2} \nabla \cdot \mathbf{u}=0
$$

Applying $D_{11}^{2} D_{12}^{2} \nabla$ to (4.1) and using (4.2), we conclude that for zero body force

$$
D_{11}^{2} D_{12}^{2} D_{21}^{2} D_{22}^{2} \nabla^{4} \mathbf{u}=0
$$

which, for the case $\varepsilon=0$, is just the well-known result that $\mathbf{u}$ is biharmonic. We are thus led to the Generalized Galerkin-Somigliana Representation

$$
\frac{2}{\kappa+1} \mathbf{u}=D_{11}^{2} D_{12}^{2} \nabla^{2} \mathbf{G}-\left[D_{11}^{2} D_{12}^{2}-\frac{\kappa-1}{\kappa+1} D_{21}^{2} D_{22}^{2}\right] \nabla \nabla \cdot \mathbf{G} .
$$

The result of substituting (4.4) into (4.1) is

$$
D_{11}^{2} D_{12}^{2} D_{21}^{2} D_{22}^{2} \nabla^{4} \mathbf{G}=-\frac{2}{\kappa+1} \mathbf{f},
$$

which, together with (4.4), completes the desired generalization.

Let the first term of (4.4) be denoted by $\mathbf{B}$, i.e.,

$$
\mathbf{B}=D_{11}^{2} D_{12}^{2} \nabla^{2} \mathbf{G} .
$$

Then, by (4.5),

$$
D_{21}^{2} D_{22}^{2} \nabla^{2} \mathbf{B}=-\frac{2}{\kappa+1} \mathbf{f} .
$$

If the objective is to reexpress (4.4) in terms of $\mathbf{B}$, the solution to a lower-order equation, the remaining terms in (4.4) must be appropriately modified. 
We begin by expressing the solution to (4.7) in terms of three component functions as follows:

$$
\begin{gathered}
\mathbf{B}=\mathbf{B}^{(0)}+\varepsilon^{2} C_{21} \mathbf{B}^{(1)}+\varepsilon^{2} C_{22} \mathbf{B}^{(2)} ; \\
\mathbf{B}^{(0)}=D_{22}^{2} D_{21}^{2} \mathbf{B}, \quad \mathbf{B}^{(2)}=D_{22}^{2} \nabla^{2} \mathbf{B}, \quad \mathbf{B}^{(2)}=D_{21}^{2} \nabla^{2} \mathbf{B} ; \\
\nabla^{2} \mathbf{B}^{(0)}=-\frac{2}{\kappa+1} \mathbf{f} ; \\
D_{21}^{2} \mathbf{B}^{(1)}=-\frac{2}{\kappa+1} \mathbf{f} ; \\
D_{22}^{2} \mathbf{B}^{(2)}=-\frac{2}{\kappa+1} \mathbf{f} ;
\end{gathered}
$$

where

$$
C_{\beta 1}=-\frac{\alpha_{\beta 1}^{4}}{\alpha_{\beta 2-}^{2} \alpha_{\beta 1}^{2}}, \quad C_{\beta 2}=\frac{\alpha_{\beta 2}^{4}}{\alpha_{\beta 2-}^{2} \alpha_{\beta 1}^{2}},
$$

in which $\beta=1,2$; and $C_{11}, C_{12}$ are defined for a similar equation to be developed in the sequel. The validity of the above decomposition may be easily verified by direct substitution (Doyle [4]).

The divergence of (4.6) is

$$
D_{11}^{2} D_{12}^{2} \nabla^{2} \nabla \cdot \mathbf{G}=\nabla \cdot \mathbf{B},
$$

which, together with the first of (4.9), yields

$$
D_{11}^{2} D_{12}^{2} \nabla^{2}\left(D_{21}^{2} D_{22}^{2} \boldsymbol{\nabla} \cdot \mathbf{G}\right)=\nabla \cdot \mathbf{B}^{(0)} .
$$

From the identities,

$$
\begin{gathered}
\nabla^{2} \mathbf{z} \cdot \mathbf{F}=\mathbf{z} \cdot \nabla^{2} \mathbf{F}+2 \boldsymbol{\nabla} \cdot \mathbf{F}, \\
D_{\alpha \beta}^{2} \mathbf{z} \cdot \mathbf{F}=\mathbf{z} \cdot D_{\alpha \beta}^{2} \mathbf{F}-2 \varepsilon^{2} \alpha_{\alpha \beta}^{2} \nabla \cdot \mathbf{F},
\end{gathered}
$$

the following representation is conceived:

$$
D_{21}^{2} D_{22}^{2} \boldsymbol{\nabla} \cdot \mathbf{G}=\frac{1}{2} \mathbf{z} \cdot \mathbf{B}^{(0)}+\frac{1}{2} B_{0},
$$

where $B_{0}$ is a new scalar function. Substituting the above into (4.15), we find that $B_{0}$ must satisfy

$$
\begin{aligned}
D_{11}^{2} D_{12}^{2} \nabla^{2} B_{0}= & \frac{2}{\kappa+1}\left[D_{11}^{2} D_{12}^{2} \mathbf{z} \cdot \mathbf{f}-2 \varepsilon^{2}\left(\alpha_{11}^{2}+\alpha_{12}^{2} D_{11}^{2}\right) \boldsymbol{\nabla} \cdot \mathbf{f}\right] \\
= & \frac{2}{\kappa+1}\left\{D_{11}^{2} D_{12}^{2}\left[\mathbf{z} \cdot \mathbf{f}-2 \varepsilon^{2}\left(\alpha_{11}^{2}+\alpha_{12}^{2}\right) \nabla \cdot \mathbf{f}\right]-2 \varepsilon^{4} \alpha_{11}^{2} C_{11} D_{12}^{2} \nabla^{2} \nabla \cdot \mathbf{f}\right. \\
& \left.-2 \varepsilon^{4} \alpha_{12}^{2} C_{12} D_{11}^{2} \nabla^{2} \nabla \cdot \mathbf{f}\right\}
\end{aligned}
$$


It follows from the above that the component representation for $B_{0}$ is:

$$
\begin{gathered}
B_{0}=B_{0}^{(0) *}+\varepsilon^{2} C_{11} B_{0}^{(1)}+\varepsilon^{2} C_{12} B_{0}^{(2)} ; \\
B_{0}^{(0) *}=D_{11}^{2} D_{12}^{2} B_{0} ; \quad B_{0}^{(1)}=D_{12}^{2} \nabla^{2} B_{0} ; \quad B_{0}^{(2)}=D_{11}^{2} \nabla^{2} B_{0} ; \\
\nabla^{2} B_{0}^{(0) *}=\frac{2}{\kappa+1}\left[\mathbf{z} \cdot \mathbf{f}-2 \varepsilon^{2}\left(\alpha_{11}^{2}+\alpha_{12}^{2}\right) \nabla \cdot \mathbf{f}\right] ; \\
D_{11}^{2} B_{0}^{(1)}=-\frac{4}{\kappa+1} \varepsilon^{2} \alpha_{11}^{2} \nabla \cdot \mathbf{f} ; \\
D_{12}^{2} B_{0}^{(2)}=-\frac{4}{\kappa+1} \varepsilon^{2} \alpha_{12}^{2} \nabla \cdot \mathbf{f} .
\end{gathered}
$$

We have thus effectively expressed the last term of (4.4) in terms of $\mathbf{B}^{(0)}$ and $B_{0}$.

Substituting (4.8) and (4.9) into (4.14), we get

$$
\nabla^{2}\left[D_{11}^{2} D_{12}^{2} \nabla \cdot \mathbf{G}-\varepsilon^{2}\left(C_{21} D_{22}^{2}+C_{22} D_{21}^{2}\right) \nabla \cdot \mathbf{B}\right]=\nabla \cdot \mathbf{B}^{(0)}
$$

which, in view of (4.16), permits the convenient representation

$$
D_{11}^{2} D_{12}^{2} \nabla \cdot \mathbf{G}=\varepsilon^{2}\left(C_{21} D_{22}^{2}+C_{22} D_{21}^{2}\right) \nabla \cdot \mathbf{B}+\frac{1}{2} \mathbf{z} \cdot \mathbf{B}^{(0)}+\frac{1}{2} B_{0}^{(0)},
$$

where $B_{0}^{(0)}$ satisfies

$$
\nabla^{2} B_{0}^{(0)}=-\mathbf{z} \cdot \nabla^{2} \mathbf{B}^{(0)}=\frac{2}{\kappa+1} \mathbf{z} \cdot \mathbf{f} .
$$

The second term on the right-hand side of (4.4) is now expressed in terms of $\mathbf{B}$ and $B_{0}^{(0)}$ by (4.26). In view of $(4.22)$ and $(4.27)$, it is convenient to decompose $B_{0}^{(0) *}$ into two parts as follows:

$$
\begin{gathered}
B_{0}^{(0) *}=\phi+B_{0}^{(0)} \\
\nabla^{2} \phi=-\frac{4}{\kappa+1} \varepsilon^{2}\left(\alpha_{11}^{2}+\alpha_{12}^{2}\right) \nabla \cdot \mathbf{f} .
\end{gathered}
$$

The final form of the Generalized Papkovich-Neuber Representation is obtained by substituting (4.6), (4.18), and (4.26) into (4.4), viz.

$$
\begin{aligned}
2 \mathbf{u}= & (\kappa+1) \mathbf{B}-\nabla \mathbf{z} \cdot \mathbf{B}^{(0)}+\frac{1}{2} \nabla\left[(\kappa-1) B_{0}-(\kappa+1) B_{0}^{(0)}\right] \\
& -\varepsilon^{2}(\kappa+1)\left(C_{21} D_{22}^{2}+C_{22} D_{21}^{2}\right) \nabla \nabla \cdot \mathbf{B},
\end{aligned}
$$

where the last term may be further simplified by (4.8)-(4.13). The most explicit form of the representation is

$$
\begin{aligned}
2 \mathbf{u}= & {\left[(\kappa+1) \mathbf{B}^{(0)}-\nabla \mathbf{z} \cdot \mathbf{B}^{(0)}-\nabla B_{0}^{(0)}\right] } \\
+ & \varepsilon^{2}(\kappa+1)\left[C_{21} \mathbf{B}^{(1)}+C_{22} \mathbf{B}^{(2)}-\left(\alpha_{21}^{2}+\alpha_{22}^{2}\right) \nabla \nabla \cdot \mathbf{B}^{(0)}\right. \\
& \left.+\frac{1}{2}\left(\frac{\kappa-1}{\kappa+1}\right) \nabla\left(\varepsilon^{-2} \phi+C_{11} B_{0}^{(1)}+C_{12} B_{0}^{(2)}\right)\right] \\
& -\varepsilon^{4}(\kappa+1) \nabla \nabla \cdot\left[C_{21} \alpha_{21}^{2} \mathbf{B}^{(1)}+C_{22} \alpha_{22}^{2} \mathbf{B}^{(2)}\right],
\end{aligned}
$$

where $\varepsilon^{-2} \phi$ is the particular solution of (4.29), as the homogeneous solution, which is harmonic, may be absorbed by $B_{0}^{(0)}$. The elasticity solution is recovered as $\varepsilon$ tends 
to zero. We note that each one of the component functions is governed by a secondorder equation. The functions $\mathbf{B}^{(0)}, B_{0}^{(0)}$, and $\phi$ are associated with the Laplacian operator and, hence, have no boundary-layer phenomena. The functions $\mathbf{B}^{(\alpha)}, B_{0}^{(\alpha)}$ $(\alpha=1,2)$ are governed by the operators $D_{\alpha \beta}^{2}$ defined by $(3.30)$ and contribute to the new boundary-layer phenomena.

For antiplane deformations defined by $u_{3}\left(z_{1}, z_{2}\right)$, we have

$$
2 u_{3}=(\kappa+1) B_{3}^{(0)}+\varepsilon^{2}(\kappa+1)\left(C_{21} B_{3}^{(1)}+C_{22} B_{3}^{(2)}\right),
$$

where $B_{3}^{(\alpha)}$ are of the boundary-layer type. For plane deformations defined by $u_{\alpha}\left(z_{1}, z_{2}\right)$, the displacements are

$$
\begin{aligned}
2 u_{\alpha}= & \kappa B_{\alpha}^{(0)}-B_{0, \alpha}^{(0)}-z_{\beta} B_{\beta, \alpha}^{(0)} \\
+ & \varepsilon^{2}(\kappa+1)\left[C_{21} B_{\alpha}^{(1)}+C_{22} B_{\alpha}^{(2)}-\left(\alpha_{21}^{2}+\alpha_{22}^{2}\right) B_{\beta, \beta \alpha}^{(0)}\right. \\
& \left.+\frac{1}{2}\left(\frac{\kappa-1}{\kappa+1}\right)\left(C_{11} B_{0, \alpha}^{(1)}+C_{12} B_{0, \alpha}^{(2)}+\varepsilon^{-2} \phi, \alpha\right)\right] \\
& -\varepsilon^{4}(\kappa+1)\left[C_{21} \alpha_{21}^{2} B_{\beta, \beta \alpha}+C_{22} \alpha_{22}^{2} B_{\beta, \beta \alpha}^{(2)}\right],
\end{aligned}
$$

where $B_{\alpha}^{(\beta)}, B_{0}^{(\beta)}$ are of the boundary-layer type. It follows that the terms $B_{0, \alpha}^{(1)}$ are expected to have the most significant contribution to the new boundary-layer phenomena. Thus, the constants $\alpha_{11}$ and $\alpha_{12}$ are also expected to be more significant than $\alpha_{21}$ and $\alpha_{22}$. We shall see that the former pair appears in all the surface energetic expressions.

5. Boundary layer along a smooth boundary. The appearance of the small parameter $\varepsilon$ in the operator $D_{\alpha \beta}^{2}$ defined by (3.30) indicates that the main difference between elasticity and cohesive elasticity lies in a boundary layer. An elasticity solution is but the interior solution of an associated cohesive elasticity problem. The self-equilibrating state $\mathbf{u}=\mathbf{u}^{(e)}$ given in (3.27) is a boundary-layer phenomenon and was discussed by Mindlin.

To fix ideas, let us consider a body with a smooth boundary defined by $\mathbf{z}=\mathbf{z}^{0}$. The following traction boundary-value problem is considered for the case $\mathbf{f} \equiv 0$ :

$$
\begin{aligned}
& \mathbf{t}=\stackrel{0}{\boldsymbol{\tau}}\left(\mathbf{z}^{0}\right), \\
& 1=\varepsilon \frac{1}{\mathbf{\tau}}\left(\mathbf{z}^{0} ; \varepsilon\right), \\
& 22=\varepsilon^{2} \boldsymbol{\tau}\left(\mathbf{z}^{0} ; \varepsilon\right),
\end{aligned}
$$

where the functions on the right-hand sides are prescribed generalized tractions, which may be specified to depend on $\varepsilon$. The explicit appearance of $\varepsilon$ in the governing equation (3.29) and surface-traction expressions (3.12)-(3.14) suggests the appropriateness of a regular perturbation solution in the form

$$
\mathbf{u}(\mathbf{z}, \varepsilon) \sim \mathbf{u}^{(0)}(\mathbf{z})+\varepsilon \mathbf{u}^{(1)}(\mathbf{z})+\cdots .
$$


Since (3.29) contains only powers of $\varepsilon^{2}$, it is clear that both $\mathbf{u}^{(0)}$ and $\mathbf{u}^{(1)}$ satisfy the ordinary elasticity equation, i.e.,

$$
\text { [Elasticity Operator] } \mathbf{u}^{(0)} \text { and } \mathbf{u}^{(1)}=0,
$$

which are of second order. The solutions to (5.5) cannot be adjusted to satisfy the three sets of boundary conditions (5.1)-(5.3). The expansion (5.4) is thus termed an outer-expansion (Cole [3], Van Dyke [26]). The appropriate boundary conditions for (5.5) can only be determined from matching. To facilitate such a computation it is necessary to set up a set of normal coordinates relative to the boundary surface $\mathbf{z}=\mathbf{z}^{0}$. Such coordinate systems are routinely used in the theory of shells.

Let $\xi_{\alpha}(\alpha=1,2)$ denote a set of Gaussian coordinates, so that the boundary surface is given by

$$
\mathbf{z}=\mathbf{z}^{0}=\mathbf{z}^{0}\left(\xi_{\alpha}\right)=\mathbf{z}^{0}\left(\xi_{1}, \xi_{2}\right) .
$$

The associated base vectors and first fundamental tensor are denoted by $\mathbf{a}_{\alpha}$ and $a_{\alpha \beta}$. In addition, a third base vector $\mathbf{a}_{3}$ is identified with the normal $\mathbf{n}$ to the surface, so that the position vector $\mathbf{z}$ becomes

$$
\mathbf{z}=\mathbf{z}^{0}\left(\boldsymbol{\xi}_{\alpha}\right)+\xi_{3} \mathbf{a}_{3}, \quad \mathbf{a}_{3}=\mathbf{n},
$$

where $\xi_{3}$ is the dimensionless distance from the surface along $\mathbf{n}$. The interior of the body, therefore, is defined by $\xi_{3}<0$.

In terms of the normal coordinates $\mathbf{a}_{i}$, the displacement vector is

$$
\mathbf{u}(\mathbf{z} ; \varepsilon)=\mathbf{u}^{*}(\xi)=u_{i}^{*}(\xi) \mathbf{a}_{1} / \sqrt{a_{i i}},
$$

so that $u_{i}^{*}$ are the physical components. The outer-expansion (5.4) is now rewritten as

$$
\mathbf{u} \sim\left[u_{j}^{*(0)}\left(\xi_{i}\right)+\varepsilon u_{j}^{*(0)}\left(\xi_{\alpha}, 0\right) \xi_{3}+\cdots\right] \mathbf{a}_{j} / \sqrt{a_{j j}},
$$

which, for small values of $\xi_{3}$, may be approximated by

$$
\begin{aligned}
\mathbf{u} \sim & \left\{\left[u_{j}^{*(0)}\left(\xi_{\alpha}, 0\right)+u_{j, 3}^{*(0)}\left(\xi_{\alpha}, 0\right) \xi_{3}+\cdots\right]\right. \\
& \left.+\varepsilon\left[u_{j}^{*(1)}\left(\xi_{\alpha}, 0\right)+u_{j, 3}^{*(1)}\left(\xi_{\alpha}, 0\right) \xi_{3}+\cdots\right]+\cdots\right\} \mathbf{a}_{j} / \sqrt{a_{j j}} .
\end{aligned}
$$

The coordinate $\xi_{3}$ is now stretched to become a boundary-layer coordinate $\xi$ defined by

$$
\xi=\xi_{3} / \varepsilon .
$$

The inner-expansion of the outer-expansion is obtained by substituting (5.11) into (5.10), and the result is

$$
\mathbf{u} \sim\left\{u_{j}^{(0)}\left(\xi_{\alpha}, 0\right)+\varepsilon\left[u_{j}^{*(1)}\left(\xi_{\alpha}, 0\right)+\xi u_{j, 3}^{*(0)}\left(\xi_{\alpha}, 0\right)\right]+\cdots\right\} \mathbf{a}_{j} / \sqrt{a_{j j}} .
$$

Let $\tau_{i j}^{*(n)}\left(\xi_{i}\right), n=0,1$, denote the physical components of the ordinary elasticity stress tensors associated with the ordinary elasticity solution $u_{i}^{*(n)}\left(\xi_{i}\right)$, i.e.,

$$
\tau_{i j}^{*}=2 \varepsilon_{i j}^{*}+\frac{3-\kappa}{\kappa-1} \delta_{i j} \varepsilon_{k k}^{*},
$$


where $\varepsilon_{i j}^{*}$ are the physical components of the ordinary strain tensor. On the surface $\xi_{3}=0$, we have

$$
\begin{aligned}
& \varepsilon_{33}^{*}=u_{3,3}^{*}\left(\xi_{\alpha}, 0\right), \\
& \varepsilon_{\alpha \alpha}^{*}=\frac{1}{\sqrt{a_{\alpha \alpha}}} u_{\alpha, \alpha}^{*}\left(\xi_{\beta}, 0\right)+\frac{1}{R_{\alpha \alpha}} u_{3}^{*}\left(\xi_{\beta}, 0\right) \quad(\alpha=1,2 ; \text { no sum }), \\
& \varepsilon_{\alpha 3}^{*}=\frac{1}{2}\left[\frac{1}{\sqrt{a_{\alpha \alpha}}} u_{3, \alpha}^{*}\left(\xi_{\beta}, 0\right)+u_{\alpha, 3}^{*}\left(\xi_{\beta}, 0\right)-\frac{1}{R_{\alpha \alpha}} u_{\alpha}^{*}\left(\xi_{\beta}, 0\right)\right],
\end{aligned}
$$

where $R_{11}, R_{22}$ are the normal radii of curvature. The curvatures are positive if the centers of curvature are on the side $\xi_{3}<0$.

The desired inner-expansion, consistent with (5.12), is

$$
\mathbf{u} \sim\left\{u_{j}^{*(0)}\left(\xi_{\alpha}, 0\right)+\varepsilon v_{j}\left(\xi_{\alpha}, \xi\right)+\cdots\right\} \mathbf{a}_{j} / \sqrt{a_{j j}},
$$

where $v_{j}$ are again physical components. The governing equations deduced from (3.29) are

$$
\begin{aligned}
& \left(1-\alpha_{11}^{2} \frac{\partial^{2}}{\partial \xi^{2}}\right)\left(1-\alpha_{12}^{2} \frac{\partial^{2}}{\partial \xi^{2}}\right) \frac{\partial^{2} v_{3}}{\partial \xi^{2}}=0, \\
& \left(1-\alpha_{21}^{2} \frac{\partial^{2}}{\partial \xi^{2}}\right)\left(1-\alpha_{22}^{2} \frac{\partial^{2}}{\partial \xi^{2}}\right) \frac{\partial^{2} v_{\alpha}}{\partial \xi^{2}}=0 .
\end{aligned}
$$

The leading terms of the displacement gradient tensors are:

$$
\begin{aligned}
E_{33}^{*} & =v_{3, \xi} ; \\
E_{3 \alpha}^{*} & =\frac{1}{2}\left[v_{\alpha, \xi}+2 \varepsilon_{\alpha_{3}}^{*(0)}-u_{\alpha, 3}^{*(0)}\right] ; \\
E_{\alpha \beta}^{*} & =\varepsilon_{\alpha \beta}^{*(0)} \\
E_{33 i}^{*} & =\frac{1}{\varepsilon} v_{i, \xi \xi} ; \\
E_{333 i}^{*} & =\frac{1}{\varepsilon^{2}} v_{i, \xi \xi \xi} ;
\end{aligned}
$$

where all components are physical and refer to the base $\mathbf{a}_{i}$.

There are more than a few nonvanishing stress components associated with the above but only the following are needed for the boundary conditions:

$$
\begin{aligned}
T_{33}^{*} & =\frac{\kappa+1}{\kappa-1} v_{3, \xi}+\gamma v_{3, \xi \xi \xi}+\frac{3-\kappa}{\kappa-1} \varepsilon_{\alpha \alpha}^{*(0)} ; \\
T_{3 \alpha}^{*}=T_{\alpha 3}^{*} & =v_{\alpha, \xi}+\frac{\gamma}{2} v_{\alpha, \xi \xi \xi}+2 \varepsilon_{\alpha 3}^{*(0)}-u_{\alpha, 3}^{*(0)} ; \\
T_{333}^{*} & =\varepsilon \alpha v_{3, \xi \xi} ; \\
T_{33 \beta}^{*} & =\varepsilon 2\left(\alpha_{3}+\alpha_{4}\right) v_{\beta, \xi \xi} ; \\
T_{3333}^{*} & =\varepsilon^{2}\left[\left(\beta_{0}+v_{3, \xi}+\beta v_{3, \xi \xi \xi}\right)+\gamma_{1} \varepsilon_{\alpha \alpha}^{*(0)}\right] ; \\
T_{333 \alpha}^{*} & =\varepsilon^{2}\left\{\left[\frac{1}{2} \gamma_{3} v_{\alpha, \xi}+2\left(\beta_{5}+\beta_{6}\right) v_{\alpha, \xi \xi \xi}\right]+\gamma_{3}\left(2 \varepsilon_{\alpha \alpha_{3}}^{*(0)}-u_{\alpha, 3}^{*(0)}\right)\right\} .
\end{aligned}
$$


Finally, the leading terms of the traction vectors are obtained from (3.12)-(3.14). They are

$$
\begin{aligned}
& \stackrel{0}{\mathbf{t}}=\left(T_{3 i}^{*}-\frac{1}{\varepsilon} T_{33 i, \xi}^{*}+\frac{1}{\varepsilon^{2}} T_{333 i, \xi \xi}^{*}\right) \mathbf{a}_{i} / \sqrt{a_{i i}}, \\
& \stackrel{1}{\mathbf{t}}=\left(T_{33 i}^{*}-\frac{1}{\varepsilon} T_{333 i, \xi}^{*}\right) \mathbf{a}_{i} / \sqrt{a_{i i}}, \\
& \stackrel{2}{\mathbf{t}}=T_{333 i}^{*} \mathbf{a} / \sqrt{a_{i i}} .
\end{aligned}
$$

The traction boundary conditions (5.1)-(5.3) are first rewritten in terms of $\xi$ and $\mathbf{a}_{i}$, i.e.,

$$
\stackrel{0}{\mathbf{t}}=\stackrel{0}{\boldsymbol{\tau}}^{*}\left(\xi_{\alpha}\right), \quad \stackrel{1}{\mathbf{t}}=\varepsilon \varepsilon^{1 *}\left(\xi_{\alpha}, \varepsilon\right), \quad \stackrel{2}{\mathbf{t}}=\varepsilon^{22 *} \boldsymbol{\tau}^{*}\left(\xi_{\alpha} ; \varepsilon\right) .
$$

The final form of the boundary conditions is

$$
\begin{gathered}
\beta_{0}+\gamma v_{3, \xi}+\beta v_{3, \xi \xi \xi}=\tau_{3}^{2 *}\left(\xi_{\alpha} ; 0\right)-\gamma_{1} \varepsilon_{\alpha \alpha}^{*(0)} \\
(\alpha-\gamma) v_{3, \xi \xi}-\beta v_{3, \xi \xi \xi \xi}=\tau_{3}^{*}\left(\xi_{\alpha} ; 0\right) \\
\frac{\kappa+1}{\kappa-1} v_{3, \xi}+(2 \gamma-\alpha) v_{3, \xi \xi \xi}+\beta v_{3, \xi \xi \xi \xi \xi}=\tau^{*}\left(\xi_{\alpha}\right)-\frac{3-\kappa}{\kappa-1} \varepsilon_{\alpha \alpha}^{*(0)}\left(\xi_{\alpha}, 0\right) \\
\frac{1}{2} \gamma_{3} v_{\lambda, \xi}+2\left(\beta_{5}+\beta_{6}\right) v_{\lambda, \xi \xi \xi}=\stackrel{2}{\tau}_{\tau}^{2 *}\left(\xi_{\beta}, 0\right)-\gamma_{3}\left(2 \varepsilon_{\lambda_{3}}^{*(0)}-u_{\lambda, 3}^{*(0)}\right) \\
{\left[2\left(\alpha_{3}+\alpha_{4}\right)-\frac{1}{2} \gamma_{3}\right] v_{\lambda, \xi \xi}-2\left(\beta_{5}+\beta_{6}\right) v_{\lambda, \xi \xi \xi \xi}=\tau_{\lambda}^{*}\left(\xi_{\beta} ; 0\right)} \\
v_{\lambda, \xi}+\left[\gamma_{3}-2\left(\alpha_{3}+\alpha_{4}\right)\right] v_{\lambda, \xi \xi \xi}+2\left(\beta_{5}+B_{6}\right) v_{\lambda, \xi \xi \xi \xi \xi} \\
=\tau_{\lambda}^{*}\left(\xi_{\beta}\right)+u_{\alpha, 3}^{*(0)}\left(\xi_{\beta}, 0\right)-2 \varepsilon_{\alpha 3}^{*(0)}\left(\xi_{\beta}, 0\right)
\end{gathered}
$$

The following identities, which are obtained from (3.31), will be used repeatedly:

$$
\begin{aligned}
\alpha_{11}^{2}+\alpha_{12}^{2} & =\frac{\kappa-1}{\kappa+1}(\alpha-2 \gamma), & \alpha_{11}^{2} \alpha_{12}^{2} & =\frac{\kappa-1}{\kappa+1} \beta ; \\
\alpha_{21}^{2}+\alpha_{22}^{2} & =2\left(\alpha_{3}+\alpha_{4}\right)-\gamma_{3}, & \alpha_{21}^{2} \alpha_{22}^{2} & =2\left(\beta_{5}+\beta_{6}\right) .
\end{aligned}
$$

Using these identities, we find that (5.24), (5.27) are, respectively, the first integrals of the governing equations (5.16), (5.17). The solutions to these equations, for $\xi<0$, are

$$
\begin{aligned}
& v_{3}=u_{3}^{*(1)}\left(\xi_{\alpha}, 0\right)+\xi u_{3,3}^{*(0)}\left(\xi_{\alpha}, 0\right)+A_{13} e^{\xi / \alpha_{11}}+B_{13} e^{\xi / \alpha_{12}} \\
& v_{\beta}=u_{\beta}^{*(1)}\left(\xi_{\alpha}, 0\right)+\xi u_{\beta, 3}^{*(0)}\left(\xi_{\alpha}, 0\right)+A_{2 \beta} e^{\xi / \alpha_{21}}+B_{2 \beta} e^{\xi / \alpha_{22}}
\end{aligned}
$$

and

$$
\begin{aligned}
u_{3,3}^{*(0)}\left(\xi_{\alpha}, 0\right) & =\frac{\kappa-1}{\kappa+1}\left[\tau_{3}^{0 *}\left(\xi_{\alpha}\right)-\frac{3-\kappa}{\kappa-1} \varepsilon_{\alpha \alpha}^{*(0)}\left(\xi_{\alpha}, 0\right)\right], \\
u_{\beta, 3}^{*(0)}\left(\xi_{\alpha}, 0\right) & =\stackrel{0}{\tau}_{\beta}^{*}\left(\xi_{\alpha}\right)+u_{\beta, 3}^{*(0)}\left(\xi_{\alpha}, 0\right)-2 \varepsilon_{\beta 3}^{*(0)}\left(\xi_{\alpha}, 0\right) ;
\end{aligned}
$$

where the last two conditions are the results of matching, i.e., the outer-expansion of the inner-expansion must match the inner-expansion of the outer-expansion given by 
(5.12). Using (5.13) and (5.15), we find from the above that the boundary conditions for the elasticity solution $u_{i}^{*(1)}\left(\xi_{j}\right)$ are just the traction conditions:

$$
\tau_{i_{3}}^{*(0)}\left(\xi_{\alpha}, 0\right)=\tau_{i}^{*}\left(\xi_{\alpha}\right),
$$

and, hence, the first term of the outer-expansion (5.9) is completely defined.

The remaining boundary conditions (5.22), (5.23), (5.25), and (5.26) involve only derivatives of $v$ and, hence, the constant terms $u_{i}^{*(1)}\left(\xi_{\alpha}, 0\right)$ cannot be determined from the inner-expansion. A lengthy calculation yields the following concise results for the other two sets of constants:

$$
\begin{aligned}
A_{\beta j} & =\frac{\left(\alpha_{\beta 0}^{2}+\alpha_{\beta 2}^{2}\right) \alpha_{\beta 1}^{2} L_{j 2}-\left(a_{\beta 0}^{2}+\alpha_{\beta 1}^{2}\right) \alpha_{\beta 1} L_{j 1}}{\left(\alpha_{\beta 0}^{2}+\alpha_{\beta 2}^{2}\right)^{2} \alpha_{\beta 1}-\left(\alpha_{\beta 0}^{2}+\alpha_{\beta 1}^{2}\right)^{2} \alpha_{\beta 2}}, \\
B_{\beta j} & =\frac{-\left(\alpha_{\beta 0}^{2}+\alpha_{\beta 1}^{2}\right) \alpha_{\beta 2}^{2} L_{j 2}+\left(\alpha_{\beta 0}^{2}+\alpha_{\beta 2}^{2}\right) \alpha_{\beta 2} L_{j 1}}{\left(\alpha_{\beta 0}^{2}+\alpha_{\beta 2}^{2}\right)^{2} \alpha_{\beta 1}-\left(\alpha_{\beta 0}^{2}+\alpha_{\beta 1}^{2}\right)^{2} \alpha_{\beta 2}},
\end{aligned}
$$

where

$$
\begin{gathered}
\alpha_{10}^{2}=\frac{\kappa-1}{\kappa+1} \gamma, \quad \alpha_{20}^{2}=\frac{1}{2} \gamma_{3} ; \\
L_{31}=\alpha_{11} \alpha_{12} \frac{\kappa-1}{\kappa+1} \tau_{3}^{*}\left(\xi_{\alpha} ; 0\right) ; \\
L_{32}=-\frac{\kappa-1}{\kappa+1} \beta_{0}-\alpha_{10}^{2} u_{3,3}^{*(0)}\left(\xi_{\alpha}, 0\right)+\frac{\kappa-1}{\kappa+1}\left[\tau_{3}^{2 *}\left(\xi_{\alpha}, 0\right)-\gamma_{1} \varepsilon_{\alpha \alpha}^{*(0)}\right] ; \\
L_{\beta 1}=\alpha_{21} \alpha_{22} \tau_{\beta}^{*}\left(\xi_{\alpha} ; 0\right) ; \\
L_{\beta 2}=-\alpha_{20}^{2} u_{\beta, 3}^{*(0)}\left(\xi_{\alpha}, 0\right)+{ }_{\tau}^{2 *}\left(\xi_{\alpha} ; 0\right)-\gamma_{3}\left(2 \varepsilon_{\beta 3}^{*(0)}-u_{\beta, 3}^{*(0)}\right) .
\end{gathered}
$$

With the exception of the terms $u_{i}^{*(1)}\left(\xi_{\alpha}, 0\right)$, which are but constants in the innerexpansion (5.15), the desired two-term inner-expansion, (5.15), is now complete.

Had we actually begun with the presumption that $\alpha_{\lambda \mu}$ are complex, (3.38) and (3.39), then $B_{\beta j}$ should be replaced by $\bar{A}_{\beta j}$ and (5.34) and (5.35) still hold, as the latter is merely the complex conjugate of the former.

6. Traction-free surface free energy. Consider now a body, of volume $V$ and bounding surface $\partial V$, that is completely free of external forces, i.e.,

$$
\mathbf{f}=\stackrel{0}{\boldsymbol{\tau}}^{*}\left(\xi_{(x)}\right)=\stackrel{1}{\boldsymbol{\tau}}^{*}\left(\xi_{1,} ; \varepsilon\right)=\stackrel{2}{\boldsymbol{\tau}}^{*}\left(\xi_{c \varepsilon} ; \varepsilon\right)=0
$$

It follows from (5.33) that the first term of the outer-expansion, $\mathbf{u}^{*(0)}(\mathbf{z})$, is identically zero and, hence,

$$
L_{32}=-\frac{\kappa-1}{\kappa+1} \beta_{0}, \quad L_{31}=L_{\beta 1}=L_{\beta 2}=0,
$$

by (5.37)-(5.40). The inner-expansion (5.5) is merely

$$
\begin{aligned}
& \mathbf{u}=\mathbf{u}^{(\mathbf{e})} \sim \varepsilon v_{3}(\xi) \mathbf{a}_{3} / \sqrt{a_{33}}, \\
& v_{3}=A_{13} e^{\xi / \alpha_{11}}+B_{13} e^{\xi / \alpha_{12}},
\end{aligned}
$$


where

$$
\begin{gathered}
A_{13}=-\frac{\kappa-1}{\kappa+1} \alpha_{11}^{2}\left(\alpha_{10}^{2}+\alpha_{12}^{2}\right) \beta_{0} / D_{1}, \\
B_{13}=\frac{\kappa-1}{\kappa+1} \alpha_{12}^{2}\left(\alpha_{10}^{2}+\alpha_{11}^{2}\right) \beta_{0} / D_{1}, \\
D_{1}=\left(\alpha_{10}^{2}+\alpha_{12}^{2}\right)^{2} \alpha_{11}-\left(\alpha_{10}^{2}+\alpha_{11}^{2}\right)^{2} \alpha_{12},
\end{gathered}
$$

which are determined by (5.34), (5.35).

The above solution is a self-equilibrating state and, hence, (3.31) and (3.32) apply. Thus,

$$
U^{*}=U_{e}^{*}=A F_{s}, \quad F_{s}=\frac{1}{2} \varepsilon \frac{\kappa-1}{\kappa+1} \beta_{0}^{2}\left(\alpha_{11}^{2}-\alpha_{12}^{2}\right) / D_{1}
$$

where $A$ is the total surface area and $F_{s}$ the free surface energy associated with the traction-free surface of a completely unloaded body. The dimensional form of $F_{s}$ is

$$
\begin{aligned}
\operatorname{Dim} . F_{s} & =\frac{(\kappa-1) b_{0}^{2}\left(\alpha_{11}^{2}-\alpha_{12}^{2}\right)}{2(\kappa+1) \mu l^{3} D_{1}} \\
& =\frac{(\kappa-1) b_{0}^{2}}{2(\kappa+1) \mu l^{3}} \frac{\alpha_{11}+\alpha_{12}}{\left(\alpha_{10}^{2}-\alpha_{11} \alpha_{12}\right)^{2}-\alpha_{11} \alpha_{12}\left(\alpha_{11}+\alpha_{12}\right)^{2}},
\end{aligned}
$$

which was first obtained by Mindlin. Thus, to the first order of magnitude, as $\varepsilon \rightarrow 0$, the surface energy associated with a regular traction-free boundary of a completely unloaded body is a material constant.

The expression (6.8) remains the same if $\alpha_{12}$ is the complex conjugate of $\alpha_{11}$, (3.34). Since $F_{s}$ must be positive, we have

$$
\left(\alpha_{10}^{2}-\alpha_{11} \alpha_{12}\right)^{2}-\alpha_{11} \alpha_{12}\left(\alpha_{11}+\alpha_{12}\right)^{2}>0
$$

a condition that must be satisfied by the new material constants.

The surface energy given by (6.8) is the energy, per unit area, associated with the formation of a new regular surface. The free end of a thin wire is not a regular surface as a whole unless it is sufficiently thick, in terms of the material scale $l$, as it is interacting with the free cylindrical surface. As to how thin is thin or how thick is thick the self-equilibrating state associated with a free cylinder must be analyzed. It goes without saying that the surface in the neighborhood of a notch (or crack) tip is not regular in the sense that $(6.8)$ is also not applicable. The conclusion is that the energy required in the formation of new surfaces is not always determined by (6.8), and many relevant self-equilibrating states must be analyzed to understand the phenomenon. In short, the energy required to scoop a smooth marble out of a chunk of solid, or slice it into halves, may be computed from (6.8). The so-called toughness may not be directly obtained from the material constant $F_{s}$.

Let us now consider a traction-free surface on a loaded body. To avoid the unnecessary complication of dividing up the bounding surface into different portions, we assume that the load is merely a body force so that the bounding surface is still completely free. The outer-expansion $\mathbf{u}^{*(0)}(\boldsymbol{\xi}),(5.9)$, is now no longer zero. Using 
the fact that $\partial V$ is traction-free, we have

$$
u_{3,3}^{*(0)}\left(\xi_{\alpha}, 0\right)=-\frac{3-\kappa}{2(7-\kappa)} \tau_{\beta \beta}^{*(0)}\left(\xi_{\alpha}, 0\right),
$$

where $\tau_{\alpha \alpha}^{*(0)}\left(\xi_{\alpha}, 0\right)$ is the surface (elasticity) stress invariant produced by the nonzero body force $\mathbf{f}$. The inner-expansion associated with the still traction-free $\partial V$ is now given by (5.15), (5.29), (5.30), (5.34), (5.35) with

$$
\begin{gathered}
L_{31}=0, \quad L_{32}=-\frac{\kappa-1}{\kappa+1} \beta_{0}+\alpha_{10}^{2} \frac{3-\kappa}{2(7-\kappa)} \tau_{\alpha \alpha}^{*(0)}\left(\xi_{\alpha}, 0\right) ; \\
L_{\beta 1}=0, \quad L_{\beta 2}=-\alpha_{20}^{2} u_{\beta, 3}^{*(0)}\left(\xi_{\alpha}, 0\right) .
\end{gathered}
$$

The total potential energy of the system may be computed from (3.26), (3.25), viz.

$$
U^{*}=-\frac{1}{2} \int_{V} \mathbf{f} \cdot \mathbf{u} d \nu+\frac{1}{2} \varepsilon^{2} \beta_{0} \int_{\partial V} \partial_{n} \nabla \cdot \mathbf{u} d a,
$$

as the surface $\partial V$ is still free. For $\varepsilon=0$, we recover the ordinary elasticity potential energy associated with the system, i.e.,

$$
\left.U^{*}\right|_{\varepsilon=0}=U_{E 1}^{*}=-\frac{1}{2} \int_{V} \mathbf{f} \cdot \mathbf{u}^{(0)} d \nu,
$$

where $\mathbf{u}^{(0)}(\mathbf{z})=\mathbf{u}^{*(0)}(\xi)$.

The second integral of $(6.14)$ is now computed to give

$$
U^{*}=U_{E 1}^{*}+A F_{s}\left(\tau_{\alpha \alpha}^{*}\right),
$$

where, to the first order of magnitude,

$$
\begin{aligned}
F_{s}\left(\tau_{\alpha \alpha}^{*}\right) & =\frac{1}{2} \varepsilon \beta_{0} v_{3,33} \\
& =\frac{1}{2} \varepsilon \beta_{0}\left[\frac{\kappa-1}{\kappa+1} \beta_{0}-\alpha_{10}^{2} \frac{3-\kappa}{2(7-\kappa)} \tau_{\alpha \alpha}^{*(0)}\left(\xi_{\alpha}, 0\right)\right]\left(\alpha_{11}^{2}-\alpha_{12}^{2}\right) / D_{1},
\end{aligned}
$$

which is to be compared with (6.8). It is noted that the contributions of $\mathbf{u}^{(0)}$ and $v_{\alpha}$ in the surface integral of (6.14) are of one order lower than that of $v_{3}$ and, hence, do not appear in (6.17). The contribution of the modulus of cohesion $\beta_{0}$ is a constant triple stress, the last term of (3.21), which gives rise to $F_{s}(0)=F_{s}$ of $(6.8)$. It follows that the interaction between $\beta_{0}$ and $\mathbf{f}$, which gives rise to $\tau_{\alpha \gamma \alpha}^{*(0)}$, is

$$
\Delta F_{s}=F_{s}\left(\tau_{\alpha \alpha}^{*(0)}\right)-F_{s}=-\frac{(3-\kappa) \gamma}{2(7-\kappa) \beta_{0}} \tau_{\alpha \alpha}^{*(0)} F_{s},
$$

which is negative if the surface stress (not to be confused with surface tension, a term that is deliberately avoided in our presentation) invariant $\tau_{\alpha(x)}^{*(0)}$ is positive. The total potential energy of the system now becomes

$$
U^{*}=U_{E 1}^{*}+A F_{s}+A\left(\Delta F_{s}\right) \text {. }
$$

Now consider an infinite slab of thickness $H$ with a cylindrical hole of radius $R$. The slab is uniformly stretched at infinity by a tensile stress $\mathbf{t}=\sigma \mathbf{e}_{r}$ while $\mathbf{e}_{r}$ is the unit radial vector. The elasticity potential energy is

$$
U_{E 1}^{*}=U_{\infty}^{*}-\frac{\pi(\kappa+1)}{4} \sigma^{2} R^{2} H
$$


where $U_{\infty}^{*}$ is the energy associated with the slab without the hole and the second term is the so-called flaw (hole) energy (Sih and Liebowitz [23]). The cohesive elasticity potential energy may be obtained from (6.16) and (6.17) with

$$
\tau_{\alpha \alpha}^{*(0)}=2 \sigma+2 \nu \sigma \doteq \frac{7-\kappa}{2} \sigma \quad \text { (plane strain) } .
$$

Thus,

$$
U^{*}=U_{E 1}^{*}+2 \pi R H\left[1-\frac{(3-\kappa) \gamma}{4 \beta_{0}} \sigma\right] F_{s},
$$

and the change of $U^{*}$ associated with a hypothetical hole expansion is

$$
\frac{\partial U^{*}}{\partial R}=-\frac{\pi(\kappa+1)}{2} H R \sigma^{2}+2 \pi H\left[1-\frac{(3-\kappa) \gamma}{4 \beta_{0}} \sigma\right] F_{s},
$$

where the first term is the elasticity potential energy release rate. The vanishing of (6.23) is the condition for the hypothetical expansion and the elasticity counterpart of such a condition is the Griffith criterion

$$
-\frac{\pi(\kappa+1)}{2} H R \sigma^{2}+2 \pi H F_{s}=0 .
$$

The relevancy or irrelevancy of the hypothetical expansion condition is not the purpose of our discussion. Rather, we use (6.23), (6.24) to illustrate the main difference between cohesive elasticity and elasticity. The surface energy term is a part of the total potential energy computed from the field solution in (6.23), whereas it is merely an appended side condition in (6.24). Moreover, the role of the interaction energy, (6.18), is apparent in (6.23).

7. Interfacial energies. Let us now assume that the upper and lower half spaces $V^{ \pm}$are taken from two different solids. We propose to glue them together, by a layer of adhesive of vanishing thickness, to form a composite body $V$. Such a process cannot be nontrivially defined by the ordinary theory of elasticity. Noting that the most striking difference between elasticity and cohesive elasticity is the existence of a constant triple stress $T_{i i j j}=\varepsilon^{2} \beta_{0},(3.21)$, we assume that the desired adhesion is the result of a singular variation in $\beta_{0}$. Moreover, the two half spaces $V^{ \pm}$are assumed to be identical in every respect other than the possibility of a difference in $\beta_{0}$. Before proceeding, it is convenient to summarize the relevant one-dimensional theory as follows:

$$
\begin{aligned}
& \left(1-\alpha_{11}^{2} \frac{d^{2}}{d \xi^{2}}\right)\left(1-\alpha_{12}^{2} \frac{d^{2}}{d \xi^{2}}\right) \frac{d^{2} v}{d \xi^{2}}+\frac{\kappa-1}{\kappa+1} \frac{d^{3} \beta_{0}}{d \xi^{3}}=0 ; \\
& \stackrel{0}{\tau}_{3}=\frac{\kappa+1}{\kappa-1}\left[\left(1-\alpha_{11}^{2} \frac{d^{2}}{d \xi^{2}}\right)\left(1-\alpha_{12}^{2} \frac{d^{2}}{d \xi^{2}}\right) \frac{d v}{d \xi}\right] ; \\
& { }_{\tau_{3}}^{1}=(\alpha-\gamma) \frac{d^{2} v}{d \xi^{2}}-\beta \frac{d^{4} v}{d \xi^{4}} ; \\
& \stackrel{2}{\tau}_{3}=\beta_{0}+\gamma \frac{d v}{d \xi}+\beta \frac{d^{3} v}{d \xi^{3}},
\end{aligned}
$$

\footnotetext{
${ }^{7}$ The stress concentration factor is 2 .
} 
where $v(\xi)$ is the $v_{3}$ of $(5.16),(5.22)-(5.24)$, and the possibility of a spatially varying $\beta_{0}$ is retained in the equation of equilibrium (7.1). The above equations are to be applied to $V^{ \pm}$defined by $\xi \gtrless 0$ in which the modulus of cohesion $\beta_{0}$ is assumed to be of different constant values $\beta_{0}^{ \pm}$. In addition, $\beta_{0}$ is assumed to have certain singular behaviors at $\xi=0$. Specifically, we assume that

$$
\beta_{0}(\xi)=\beta_{0}^{-}+\left(\beta_{0}^{+}-\beta_{0}^{-}\right) \delta_{0}(\xi)+\beta_{0}^{*} \delta(\xi),
$$

where $\delta_{0}(\xi)$ and $\delta(\xi)$ are, respectively, the unit step function and delta function, such that

$$
\frac{d}{d \xi} \delta_{0}(\xi)=\delta(\xi), \quad \int_{-\infty}^{+\infty} \delta(\xi) d \xi=1
$$

The constant $\beta_{0}^{*}$ is a new parameter characterizing the adhesion. For convenience, it is defined in terms of yet another parameter $\beta_{0}^{A}$, the modulus of cohesion of the adhesive material, by the expression

$$
\beta_{0}^{*}=\frac{\left(\alpha_{11}+\alpha_{12}\right) \alpha_{11}^{2} \alpha_{12}^{2}}{\left(\alpha_{10}^{2}-\alpha_{11} \alpha_{12}\right)^{2}-\alpha_{11} \alpha_{12}\left(\alpha_{11}+\alpha_{12}^{2}\right)} \beta_{0}^{A},
$$

where the dimension of the dimensional form of the factor, which is introduced for convenience (c.f. (6.9), (6.10), (3.34)), is $l$ and

$$
\text { Dim. } \beta_{0}^{A}=b_{0}^{A}=\mu l^{2} \beta_{0}^{A},
$$

so that the interpretation of $(7.5)$ is dimensionally correct.

The solution satisfying (7.1) and ${ }_{\tau_{3}^{ \pm}}^{ \pm}(0)=0$ is

$$
v^{ \pm},{ }_{\xi}(\xi)=A^{ \pm} e^{\mp \xi / \alpha_{11}}+B^{ \pm} e^{\mp \xi / \alpha_{12}} \quad(\xi \gtrless 0),
$$

where the four constants $A^{ \pm}, B^{ \pm}$must now be determined to meet the implication of (7.5). It follows from all the above relations and additional continuity requirements that

$$
\begin{gathered}
v^{+},{ }_{\xi \xi \xi}(0)-v^{-},{ }_{\xi \xi \xi}(0)=-\frac{1}{\beta}\left(\beta_{0}^{+}-\beta_{0}^{-}\right) ; \\
v^{+},{ }_{\xi \xi}(0)-v^{-},{ }_{\xi \xi}(0)=-\frac{1}{\beta} \beta_{0}^{*} ; \\
v^{+},{ }_{\xi}(0)=v^{-},{ }_{\xi}(0) ; \\
1_{3}^{+}(0)=\tau_{3}^{-}(0) ; \\
2_{3}^{+}(0)=\tau_{3}^{-}(0) \quad \text { (identity), }
\end{gathered}
$$

where the last condition is identically satisfied if $(7.10)$ and $(7.12)$ are met. It is also clear from (7.13) and (7.2) that, in addition to (7.10) and (7.11), the 4th and 5th derivatives of $v$ are also discontinuous, even though the generalized tractions are all continuous. The condition (7.12) conforms with the ordinary elasticity requirement, as there is no concentrated force in the problem. 
The four constants obtained from the application of (7.10)-(7.13) are

$$
\begin{gathered}
A^{ \pm}=\frac{(\kappa-1)}{2(\kappa+1)}-\left[\frac{\left(\alpha_{10}^{2}+\alpha_{12}^{2}\right) \alpha_{11}}{D_{1}} \beta_{0}^{A} \mp \frac{\beta_{0}^{+}-\beta_{0}^{-}}{\alpha_{12}^{2}-\alpha_{11}^{2}}\right], \\
B^{ \pm}=\frac{(\kappa-1)}{2(\kappa+1)}\left[\frac{\left(\alpha_{10}^{2}+\alpha_{11}^{2}\right) \alpha_{12}}{D_{1}} \beta_{0}^{A} \mp \frac{\beta_{0}^{+}-\beta_{0}^{-}}{\alpha_{12}^{2}-\alpha_{11}^{2}}\right],
\end{gathered}
$$

where $D_{1}$ is given by (6.7). The combination of (7.9) is a self-equilibrating state, as far as the composite body $V$ is concerned, and (3.26) gives the potential energy for the system

$$
\begin{gathered}
U^{*}=A \Gamma_{I}, \\
\Gamma_{I}=\varepsilon \frac{(\kappa-1)}{4(\kappa+1)}\left[\frac{\left(\alpha_{11}^{2}-\alpha_{12}^{2}\right)\left(\beta_{0}^{+}+\beta_{0}^{-}\right) \beta_{0}^{A}}{D_{1}}-\frac{\left(\beta_{0}^{+}-\beta_{0}^{-}\right)^{2}}{\alpha_{11} \alpha_{12}\left(\alpha_{11}+\alpha_{12}\right)}\right],
\end{gathered}
$$

where $\Gamma_{I}$ is the interfacial free energy. Since the interfacial free energy must be nonnegative, we have

$$
\beta_{0}^{A} \geq \beta_{0 m}^{A} \equiv\left[\frac{\left(\alpha_{10}^{2}-\alpha_{11} \alpha_{12}\right)^{2}}{\left(\alpha_{11}+\alpha_{12}\right)^{2} \alpha_{11} \alpha_{12}}-1\right] \frac{\left(\beta_{0}^{+}-\beta_{0}^{-}\right)^{2}}{\beta_{0}^{+}+\beta_{0}^{-}},
$$

where the right-hand side is positive by (6.10).

The energy of adhesion, $E_{\mathrm{Ad}}$, is defined as the amount of work necessary to increase the separation of two surfaces from zero to infinite distance, and is defined by (Murr [22])

$$
E_{\mathrm{Ad}}=F_{s}^{+}+F_{s}^{-}-\Gamma_{I},
$$

where $F_{s}^{ \pm}$are computed by (6.8). The energy of adhesion must also be nonnegative. Thus,

$$
\beta_{0}^{A} \leq \beta_{0 M}^{A} \equiv \beta_{0}^{+}+\beta_{0}^{-}+\frac{\left(\alpha_{10}^{2}-\alpha_{11} \alpha_{12}\right)^{2}}{\left(\alpha_{11}+\alpha_{12}\right)^{2} \alpha_{11} \alpha_{12}}-\frac{\left(\beta_{0}^{+}-\beta_{0}^{-}\right)^{2}}{\left(\beta_{0}^{+}+\beta_{0}^{-}\right)} .
$$

In summary, we have

$$
\beta_{0 m}^{A} \leq \beta_{0}^{A} \leq \beta_{0 M}^{A}
$$

Perfect Adhesion:

$$
\beta_{0}^{A}=\beta_{0 m}^{A}, \quad \Gamma_{I}=0, \quad E_{\mathrm{Ad}}=F_{s}^{+}+F_{s}^{-} ;
$$

Adhesionless Adhesion:

$$
\beta_{0}^{A}=\beta_{0 M}^{A}, \quad \Gamma_{I}=F_{s}^{+}+F_{s}, \quad E_{\mathrm{Ad}}=0 .
$$

For the case of identical $V^{ \pm}$, i.e., $\beta_{0}^{+}=\beta_{0}^{-}=\beta_{0}$, we have

$$
0=\beta_{0 m}^{A} \leq \beta_{0}^{A} \leq \beta_{0 M}^{A}=2 \beta_{0} .
$$

It is seen from (7.9), (7.15), and (7.16) that

$$
\begin{gathered}
v,_{\xi} \equiv 0 \quad \text { for } \beta_{0}^{+}=\beta_{0}^{-}=\beta_{0}, \beta_{0}^{A}=0 ; \\
v,_{\xi}=v_{3, \xi} \text { of }(6.4), \text { for } \beta_{0}^{+}=\beta_{0}^{-}=\beta_{0}, \beta_{0}^{A}=2 \beta_{0} .
\end{gathered}
$$


The last condition indicates that the traction-free solution is recovered via the singularity representation (7.5) without the specific stipulation that the generalized tractions $\stackrel{1}{\tau}_{3}$ and $\stackrel{2}{\tau}_{3}$ be zero. In this connection, it is more convenient to interpret the singular $\beta_{0}$-variation of (7.1) as the body force, i.e.,

$$
f(\xi)=\frac{d^{3} \beta_{0}}{d \xi^{3}} .
$$

Thus, the solution to two disjointed half spaces, in the sense of (7.27), may be obtained from the solution to a full space subjected to a suitably defined singular body force. This observation opens up the possibility of defining a microcrack via the introduction of singular body force systems. A crack is termed a microcrack if its dimensionless length is of the order of $\varepsilon$ and, as a result, every term of the governing equation (4.1) is of the same order, as the independent variable $\mathbf{z}$ must be rescaled by $\varepsilon$. The explicit component-function representation obtained in Sect. 4, together with the above body-force observation, lead us to believe that an explicit solution for the microcrack problem is an achievable goal. This problem is being pursued by us.

In terms of cohesive elasticity, which is still a crude continuum model for solids, a grain boundary or an interphase-interface is but a special adhesive boundary. Thus, different physical interpretations could be assigned to the quantities $\beta_{0}^{A}, \Gamma_{1}$, and $E_{\mathrm{Ad}}$. When a grain boundary meets a free surface, a thermal groove profile for grain boundary-surface equilibrium may be induced. If the angle sustained by the groove is $\Omega_{s}$, then the grain boundary free energy $\Gamma_{\mathrm{gb}}$, which may be identified with $\Gamma_{1}$ of (7.8), is

$$
\Gamma_{\mathrm{gb}}=2 F_{s} \operatorname{Cos}\left(\Omega_{s} / 2\right) .
$$

The groove configuration is a singular boundary layer phenomenon, in terms of our analytical treatment, and must be analyzed separately. It is now also possible to refine the mathematical definition of an interfacial crack-another class of singular problems to be solved.

8. Thin film energy and the concept of an interface-phase. A layer of solid is called a plate if its thickness is much larger than the material length scale $l$. It is termed a film if its dimensionless thickness is of the order of $\varepsilon$. The governing equations for a free film are just (7.1)-(7.4), and the boundary conditions are

$$
\stackrel{0}{\tau}_{3}=\stackrel{1}{\tau}_{3}=\stackrel{2}{\tau}_{3}=0 \quad \text { at } \xi= \pm h,
$$

where $2 h$ is the dimensionless thickness. The solution is

$$
\begin{aligned}
v(\xi)=\frac{(\kappa-1) \beta_{0}}{(\kappa+1) D_{1}(h)}\left[-\alpha_{11}^{2}\left(\alpha_{10}^{2}+\alpha_{12}^{2}\right)\right. & \operatorname{Sinh} \frac{h}{\alpha_{12}} \operatorname{Sinh} \frac{\xi}{\alpha_{11}} \\
& \left.+\alpha_{12}^{2}\left(\alpha_{10}^{2}+\alpha_{11}^{2}\right) \operatorname{Sinh} \frac{h}{\alpha_{11}} \operatorname{Sinh} \frac{\xi}{\alpha_{12}}\right],
\end{aligned}
$$

where

$$
D_{1}(h)=\alpha_{11}\left(\alpha_{10}^{2}+\alpha_{12}^{2}\right)^{2} \operatorname{Cosh} \frac{h}{\alpha_{11}} \operatorname{Sinh} \frac{h}{\alpha_{12}}-\alpha_{12}\left(\alpha_{10}^{2}+\alpha_{11}^{2}\right)^{2} \operatorname{Cosh} \frac{h}{\alpha_{12}} \operatorname{Sinh} \frac{h}{\alpha_{11}} \text {, }
$$


which is to be compared with $D_{1}$ of $(6.3)-(6.7)$.

The above solution is self-equilibrating, and (3.27) still applies. The total potential energy for the system is

$$
\begin{gathered}
U^{*}=A F_{F}, \\
F_{F}=\varepsilon \frac{\kappa-1}{\kappa+1} \beta_{0}^{2}\left(\alpha_{11}^{2}-\alpha_{12}^{2}\right) \operatorname{Sinh} \frac{h}{\alpha_{11}} \operatorname{Sinh} \frac{h}{\alpha_{12}} / D_{1}(h),
\end{gathered}
$$

where $F_{F}$ is the film free energy, per unit area of the film, which is to be compared with (6.8). We have

$$
\lim _{h \rightarrow \infty} F_{F}(h)=2 F_{s},
$$

where $F_{s}$ is the free-surface free energy (6.8). The limit simply states the fact that the free surfaces of a (thick) plate do not interact. As $h$ tends to zero, we have

$$
\lim _{h \rightarrow 0} F_{F}(h)=\varepsilon \frac{\kappa-1}{\kappa+1} \beta_{0}^{2} h /\left(\alpha_{10}^{4}-\alpha_{11}^{2} \alpha_{12}^{2}\right),
$$

which is to be interpreted as the mathematical approximation for $F_{F}(h)$, for small values of $h$. Since $F_{F}$ must be nonnegative, we have (c.f. (3.34))

$$
\alpha_{10}^{2}>\alpha_{11} \alpha_{12} \text {. }
$$

It follows from the above that (6.10) may be replaced by

$$
\alpha_{10}^{2}-\alpha_{11} \alpha_{12}>\left(\alpha_{11}+\alpha_{12}\right)\left(\alpha_{11} \alpha_{12}\right)^{1 / 2} \text {. }
$$

Here we see that material-parameter properties are not solely governed by the positive definiteness of the strain energy density (c.f. the paragraph before (3.34)). It is anticipated that many, many more conditions of the kind will eventually come out of many different physical situations. It can be easily checked that $F_{F}$ is a monotonically increasing function of $h$.

Gluing two half spaces and a layer together, the layer in between is termed an interface-phase if its dimensionless thickness is of the order of $\varepsilon$. Let us assume that the two half spaces are identical, so that only half of the symmetric problem needs to be analyzed. The set of equations (7.1)-(7.4) still applies, and the lower half of the problem is defined by

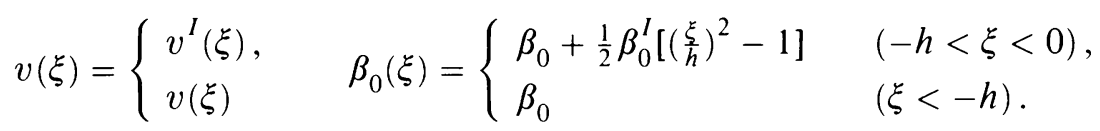

We still keep the simple assumption that all the other material properties are the same for both the half spaces and the layer.

The associated self-equilibrating solution is

$$
\begin{array}{cc}
v^{I}{ }_{\xi}(\xi)=\frac{A}{\alpha_{11}} \operatorname{Cosh} \frac{\xi}{\alpha_{11}}+\frac{B}{\alpha_{12}} \operatorname{Cosh} \frac{\xi}{\alpha_{12}}+\frac{\kappa-1}{\kappa+1} \beta_{0}^{I} / h^{2} \quad(-h<\xi<0), \\
v,_{\xi}(\xi)=\frac{A_{0}}{\alpha_{11}} e^{(\xi+h) / \alpha_{11}}+\frac{B_{0}}{\alpha_{12}} e^{(\xi+h) / \alpha_{12}} & (\xi<-h),
\end{array}
$$


where

$$
\begin{array}{rlrl}
A_{0}=-A \operatorname{Sinh} \frac{h}{\alpha_{11}}, & B_{0} & =-B \operatorname{Sinh} \frac{h}{\alpha_{12}} \\
A=A^{*} /\left(\operatorname{Cosh} \frac{h}{\alpha_{11}}+\operatorname{Sinh} \frac{h}{\alpha_{11}}\right), & B & =B^{*} /\left(\operatorname{Cosh} \frac{h}{\alpha_{12}}+\operatorname{Sinh} \frac{h}{\alpha_{12}}\right) ; \\
A^{*}=\frac{\alpha_{11}^{3}}{\alpha_{12}^{2}-\alpha_{11}^{2}} \frac{\kappa-1}{\kappa+1} \frac{\beta_{0}^{I}}{h^{2}}, & B^{*}=-\frac{\alpha_{12}^{3}}{\alpha_{12}^{2}-\alpha_{11}^{2}} \frac{\kappa-1}{\kappa+1} \frac{\beta_{0}^{I}}{h^{2}}
\end{array}
$$

The total potential energy of the system is again given by (3.27) except that $\beta_{0}(\xi)$ is now the function defined by (8.11). We have

$$
\begin{aligned}
& U^{*}=A \Gamma_{I I}, \\
& \times\left\{\frac{\alpha_{12}^{2}\left(h \operatorname{Cosh} \frac{h}{\alpha_{12}}-\alpha_{12} \operatorname{Sinh} \frac{h}{\alpha_{11}}\right)}{\operatorname{Sinh} \frac{h}{\alpha_{12}}+\operatorname{Cosh} \frac{h}{\alpha_{12}}}-\frac{\alpha_{11}^{2}\left(h \operatorname{Cosh} \frac{h}{\alpha_{11}}-\alpha_{11} \operatorname{Sinh} \frac{h}{\alpha_{11}}\right)}{\operatorname{Sinh} \frac{h}{\alpha_{11}}+\operatorname{Cosh} \frac{h}{\alpha_{11}}}\right\},
\end{aligned}
$$

where $\Gamma_{I I}$ is the interface-phase free energy, per unit area of the interface, which is to be compared with $\Gamma_{I}$ of (7.18) where the interface-phase was taken as an idealized dividing surface. We have

$$
\lim _{h \rightarrow 0} \Gamma_{I I}(h)=\varepsilon\left(\frac{\kappa-1}{\kappa+1}\right)\left(\beta_{0}^{I}\right)^{2} / 3 \alpha_{11} \alpha_{12}\left(\alpha_{11}+\alpha_{12}\right),
$$

which is to be taken as the mathematical limit of $\Gamma_{I I}$, for small values of $h$. It is noted from (8.17) and (8.18) that $\Gamma_{I I}$ is always positive, regardless of the sign in front of $\beta_{0}^{I}$ in (8.10). In other words, $\Gamma_{I I}$ remains the same for

$$
\beta_{0}(\xi)=\beta_{0}-\frac{1}{2} \beta_{0}^{I}\left[\left(\frac{\xi}{h}\right)^{2}-1\right] \quad(-h<\xi<0) .
$$

The difference between the above and (8.11) lies in the fact that

$$
\begin{array}{lll}
\beta_{0}(\xi)<\beta_{0}, & \beta_{0}(0)=\beta_{0}-\frac{1}{2} \beta_{0}^{I} & \text { for }(8.11), \\
\beta_{0}(\xi)>\beta_{0}, & \beta_{0}(0)=\beta_{0}+\frac{1}{2} \beta_{0}^{I} & \text { for }(8.19) .
\end{array}
$$

The strength of such a joint is dictated by the minimum value of $\beta_{0}(\xi)$.

The $\beta_{0}(\xi)$ variation defined by $(8.10)$ is arbitrary. It is used to illustrate the meaning of an interface-phase. However, it is clear that $\beta_{0}(\xi)$ may be required to yield a minimum for $\Gamma_{I I}$. This proposition is not pursued further in this paper. It is also apparent that the energy associated with a film deposited on top of a substrate can also be meaningfully defined. 
9. Stretching of thin films. Consider now a (thin) film occupying the region

$$
\left|z_{3}\right|<\varepsilon h \text { or }|\xi|<h,
$$

where $\xi=z_{3} / \varepsilon$. The film is biaxially stretched, so that

$$
u_{1}=\varepsilon_{11} z_{1}, \quad u_{2}=\varepsilon_{22} z_{2}, \quad u_{3}=\varepsilon v(\xi),
$$

where $\varepsilon_{11}$ and $\varepsilon_{22}$ are the constant elasticity strains. For convenience, we define $\tau_{11}$ and $\tau_{22}$ by

$$
\begin{aligned}
\tau_{11} & =\frac{\kappa+1}{\kappa-1} \varepsilon_{11}+\frac{3-\kappa}{\kappa-1} \varepsilon_{22}, \\
\tau_{22} & =\frac{\kappa+1}{\kappa-1} \varepsilon_{22}+\frac{3-\kappa}{\kappa-1} \varepsilon_{11},
\end{aligned}
$$

which are obtained by substituting $\varepsilon_{11}, \varepsilon_{22}, \varepsilon_{33}=0$ into the ordinary stress-strain relation with $\kappa$ being the first expression (not the plane-stress version) of (3.18).

In terms of cohesive elasticity, the nonzero components of the strains are

$$
\begin{gathered}
E_{11}=\varepsilon_{11}, \quad E_{22}=\varepsilon_{22}, \quad E_{33}=v,_{\xi} \\
E_{333}=\frac{1}{\varepsilon} v,_{\xi \xi} ; \quad E_{333}=\frac{1}{\varepsilon^{2}} v,_{\xi \xi \xi}
\end{gathered}
$$

The nonzero components of the stresses are

$$
\begin{aligned}
& T_{\alpha \alpha}=\tau_{\alpha \alpha}+\frac{3-\kappa}{\kappa-1} v,_{\xi}+\gamma_{1} v,{ }_{\xi \xi \xi} \quad(\alpha=1,2 ; \text { no sum }) ; \\
& T_{33}=\frac{3-\kappa}{\kappa-1}\left(\varepsilon_{11}+\varepsilon_{22}\right)+\frac{\kappa+1}{\kappa-1} v,_{\xi}+\gamma v,{ }_{\xi \xi \xi} ; \\
& T_{333}=\varepsilon \alpha v,{ }_{\xi \xi} ; \\
& T_{311}=T_{131}=T_{322}=T_{232}=\varepsilon\left(\alpha_{1}+\frac{1}{2} \alpha_{2}\right) v, \xi \xi ; \\
& T_{3333}=\varepsilon^{2}\left[\left(\beta_{0}+\gamma v,{ }_{\xi}+\beta v,{ }_{\xi \xi \xi}\right)+\gamma_{1}\left(\varepsilon_{11}+\varepsilon_{22}\right)\right] ; \\
& T_{\alpha \beta 33}=\varepsilon^{2}\left\{\frac{1}{3}\left[\gamma_{1}\left(\varepsilon_{11}+\varepsilon_{22}\right) \delta_{\alpha \beta}+\gamma_{2} \varepsilon_{\alpha \beta}\right]\right. \\
& \left.+\frac{1}{3}\left[\beta_{0}+\left(\gamma_{1}+\gamma_{3}\right) v,_{\xi}+\left(2 \beta_{1}+\beta_{3}+2 \beta_{4}+2 \beta_{5}\right) v,_{\xi \xi \xi}\right] \delta_{\alpha \beta}\right\} ; \\
& T_{33 \alpha \beta}=\varepsilon^{2}\left\{\frac{1}{3}\left[\gamma_{1}\left(\varepsilon_{11}+\varepsilon_{22}\right) \delta_{\alpha \beta}+\gamma_{3} \varepsilon_{\alpha \beta}\right]\right. \\
& \left.+\frac{1}{3}\left[\beta_{0}+\left(\gamma_{1}+\gamma_{2}\right) v,_{\xi}+\left(2 \beta_{1}+2 \beta_{2}+\beta_{3}\right) v,{ }_{\xi \xi \xi}\right] \delta_{\alpha \beta}\right\} ; \\
& T_{\alpha \beta \gamma \delta}=\varepsilon^{2}\left\{\frac{1}{3} \gamma_{1}\left(\varepsilon_{11}+\varepsilon_{22}\right) \delta_{\alpha \beta \gamma \delta}+\frac{1}{3} \gamma_{2}\left(\varepsilon_{\alpha \beta} \delta_{\gamma \delta}+\varepsilon_{\alpha \gamma} \delta_{\beta \delta}+\varepsilon_{\beta \gamma} \delta_{\alpha \delta}\right)\right. \\
& +\frac{1}{3} \gamma_{3}\left(\delta_{\alpha \beta} \varepsilon_{\gamma \delta}+\delta_{\alpha \gamma} \varepsilon_{\beta \delta}+\delta_{\beta \gamma} \varepsilon_{\alpha \delta}\right) \\
& \left.+\frac{1}{3}\left[\beta_{0}+\gamma_{1} v,{ }_{\xi}+2 \beta_{1} v,{ }_{\xi \xi \xi}\right] \delta_{\alpha \beta \gamma \delta}\right\} \text {. }
\end{aligned}
$$


The governing equation for $v$ is just (7.1), and the traction-free conditions on $\xi= \pm h$ are

$$
\begin{gathered}
\frac{\kappa+1}{\kappa-1}\left[\left(1-\alpha_{11}^{2} \frac{d^{2}}{d \xi^{2}}\right)\left(1-\alpha_{12}^{2} \frac{d^{2}}{d \xi^{2}}\right) \frac{d v}{d \xi}\right]+\frac{3-\kappa}{\kappa-1} \varepsilon_{\alpha \alpha}=0, \\
(\alpha-\gamma) \frac{d^{2} v}{d \xi^{2}}-\beta \frac{d^{4} v}{d \xi^{4}}=0 \\
\gamma_{1} \varepsilon_{\alpha \alpha \alpha}+\beta_{0}+\gamma \frac{d v}{d \xi}+\beta \frac{d^{3} v}{d \xi^{3}}=0 .
\end{gathered}
$$

The solution is

$$
v=-\frac{3-\kappa}{\kappa+1} \varepsilon_{\alpha \alpha \alpha} \xi+A \operatorname{Sinh} \frac{\xi}{\alpha_{11}}+B \operatorname{Sinh} \frac{\xi}{\alpha_{12}},
$$

where

$$
\begin{aligned}
& A=-\frac{\kappa-1}{\kappa+1} \alpha_{11}^{2}\left(\alpha_{10}^{2}+\alpha_{12}^{2}\right) \operatorname{Sinh} \frac{h}{\alpha_{12}}\left[\beta_{0}+\left(\gamma_{1}-\frac{3-\kappa}{\kappa+1} \gamma\right) \varepsilon_{\alpha \alpha}\right] / D_{1}(h), \\
& B=\frac{\kappa-1}{\kappa+1} \alpha_{12}^{2}\left(\alpha_{10}^{2}+\alpha_{11}^{2}\right) \operatorname{Sinh} \frac{h}{\alpha_{11}}\left[\beta_{0}+\left(\gamma_{1}-\frac{3-\kappa}{\kappa+1} \gamma\right) \varepsilon_{\alpha \alpha}\right] / D_{1}(h),
\end{aligned}
$$

in which $D_{1}(h)$ is just the expression given by (8.3). The solution reduces to (8.2), which was obtained for a film with a fixed edge, when $\varepsilon_{\alpha x}=0$.

On the cross-section $z_{1}=$ constant, the stress-traction is

$$
\stackrel{0}{\mathbf{t}}=t_{11} \mathbf{e}_{1}, \quad t_{11}(\xi)=T_{11}-2 T_{311,3}+3 T_{3311,33},
$$

which follows from (3.12). It is noted that the stress-traction is no longer independent of the thickness coordinate. We have

$$
\begin{gathered}
t_{11}(\xi)=\tau_{11}+\tau(\xi), \\
\tau(\xi)=\frac{3-\kappa}{\kappa-1} v,_{\xi}+\left(2 \gamma_{1}+\gamma_{2}-2 \alpha_{1}-\alpha_{2}\right) v,_{\xi \xi \xi}+\left(2 \beta_{1}+\beta_{2}+\beta_{3}\right) v,_{\xi \xi \xi \xi \xi} .
\end{gathered}
$$

Similarly, the stress-traction on the cross-section $z_{2}=$ constant is

$$
\begin{aligned}
& \stackrel{0}{\mathbf{t}}=t_{22} \mathbf{e}_{2}, \quad t_{22}(\xi)=T_{22}-2 T_{322,3}+3 T_{3322,33}, \\
& t_{22}(\xi)=\tau_{22}+\tau(\xi) \text {. }
\end{aligned}
$$

The average of $\tau(\xi)$ over the thickness is

$$
\begin{aligned}
\langle\tau\rangle & =\frac{1}{2 h} \int_{-h}^{h} \tau(\xi) d \xi \\
& =\frac{1}{h}\left[\frac{3-\kappa}{\kappa-1} v(h)+\left(2 \gamma_{1}+\gamma_{2}-2 \alpha_{1}-\alpha_{2}\right) \frac{d^{2} v(h)}{d \xi^{2}}+\left(2 \beta_{1}+2 \beta_{2}+\beta_{3}\right) \frac{d^{4} v(h)}{d \xi^{4}}\right] \\
& =-\frac{(3-\kappa)^{2}}{\kappa^{2}-1} \varepsilon_{\gamma \alpha x}-\left[\beta_{0}+\left(\gamma_{1}-\frac{3-\kappa}{\kappa+1} \gamma\right) \varepsilon_{\gamma \alpha \alpha}\right] F(h),
\end{aligned}
$$


where

$$
\begin{aligned}
F(h)= & \frac{\kappa-1}{\kappa+1}\left[\frac{3-\kappa}{\kappa+1} \gamma-\left(2 \gamma_{1}+\gamma_{2}-2 \alpha_{1}-\alpha_{2}\right)-\left(2 \beta_{1}+2 \beta_{2}+\beta_{3}\right)(\alpha-\gamma) / \beta\right] \\
& \times\left(\alpha_{11}^{2}-\alpha_{12}^{2}\right) \operatorname{Sinh} \frac{h}{\alpha_{11}} \operatorname{Sinh} \frac{h}{\alpha_{12}} / h D_{1}(h) .
\end{aligned}
$$

The averaged forms of (9.20) and (9.23) are

$$
\left\langle t_{11}\right\rangle=\tau_{11}+\langle\tau\rangle, \quad\left\langle\tau_{22}\right\rangle=\tau_{22}+\langle\tau\rangle
$$

A necessary condition for a film of finite size to be completely free is $\left\langle t_{11}\right\rangle=\left\langle t_{22}\right\rangle=0$. It follows that there exists an $\varepsilon^{0}$ such that

$$
\begin{gathered}
\varepsilon_{11}=\varepsilon_{22}=\varepsilon^{0}, \quad \tau_{11}=\tau_{22}=\frac{4}{\kappa-1} \varepsilon^{0}, \\
\frac{2(7-\kappa)}{\kappa+1} \varepsilon^{0}-\left[\beta_{0}+\left(\gamma_{1}-\frac{3-\kappa}{\kappa+1} \gamma\right) 2 \varepsilon^{0}\right] F(h)=0,
\end{gathered}
$$

where the last condition is merely the requirement that $\left\langle t_{11}\right\rangle=\left\langle t_{22}\right\rangle=0$. The associated solution, therefore, is self-equilibrating, and (3.27) again applies. The total potential energy of the system is

$$
U^{*}=A F_{F}^{*}, \quad F_{F}^{*}=\frac{1}{\beta_{0}}\left[\beta_{0}+\left(\gamma_{1}-\frac{3-\kappa}{\kappa+1} \gamma\right) 2 \varepsilon^{0}\right] F_{F},
$$

where $F_{F}$ is the film free energy associated with a film with a fixed edge, (8.5). The new film free energy $F_{F}^{*}$ is associated with a film with a free edge. It must be nonnegative and also less than $F_{F}$, and hence

$$
-\beta_{0}<\left(\gamma_{1}-\frac{3-\kappa}{\kappa+1} \gamma\right) 2 \varepsilon^{0}<0
$$

It can be shown that for positive $\beta_{0}$, which is presumed throughout this paper, a material volume contracts in the presence of a traction-free surface. For example, $v, \xi$ of (8.2) is negative. It follows that $\varepsilon^{0}<2$ which, together with the second inequality of $(9.30)$, implies that

$$
\gamma_{1}-\frac{3-\kappa}{\kappa+1} \gamma=\frac{2(\kappa-1)}{\kappa+1} \gamma_{1}-\frac{3-\kappa}{\kappa+1}\left(\gamma_{2}+\gamma_{3}\right)>0
$$

The first of (9.30) and (9.28) leads to the conclusion that

$$
F(h)=\left[\frac{3-\kappa}{\kappa+1} \gamma-\left(2 \gamma_{1}+\gamma_{2}-2 \alpha_{1}-\alpha_{2}\right)-\left(2 \beta_{1}+2 \beta_{2}+\beta_{3}\right)(\alpha-\gamma) / \beta\right] F_{F} / \varepsilon \beta_{0}^{2}<0,
$$

if

$$
1<\kappa<3
$$

which is a condition for the ordinary theory of elasticity. Since $F_{F}$ is positive, we have

$$
\frac{3-\kappa}{\kappa+1} \gamma-\left(2 \gamma_{1}+\gamma_{2}-2 \alpha_{1}-\alpha_{2}\right)-\left(2 \beta_{1}+2 \beta_{2}+\beta_{3}\right)(\alpha-\gamma) / \beta<0 .
$$


A unidirectional tension test, in the $z_{1}$-direction, performed on the free film is defined by

$$
\begin{gathered}
\varepsilon_{11}=\varepsilon^{0}+e_{11}, \quad \varepsilon_{22}=\varepsilon^{0}+e_{22}, \\
\left\langle t_{22}\right\rangle=\frac{\kappa+1}{\kappa-1} e_{22}+\frac{3-\kappa}{\kappa-1} e_{11}-\left[\frac{(3-\kappa)^{2}}{\kappa^{2}-1}+\left(\gamma_{1}-\frac{3-\kappa}{\kappa+1} \gamma\right) F(h)\right] e_{\alpha \alpha}=0, \\
\left\langle t_{11}\right\rangle=2\left(e_{11}-e_{22}\right),
\end{gathered}
$$

where (9.36) has been used in defining $\left\langle t_{11}\right\rangle$. The vanishing of $\left\langle t_{22}\right\rangle,(9.36)$, yields

$$
e_{22}=-\frac{3-\kappa}{4} G(h)
$$

where

$$
G(h)=\left[1-\frac{\kappa+1}{2(3-\kappa)}\left(\gamma_{1}-\frac{3-\kappa}{\kappa+1} \gamma\right) F(h)\right] /\left[1-\frac{\kappa+1}{8}\left(\gamma_{1}-\frac{3-\kappa}{\kappa+1} \gamma\right) F(h)\right] .
$$

It follows from (9.31), (9.32), (9.33), and (9.25) that

$$
G(h)>1, \quad \lim _{h \rightarrow \infty} G(h)=1 .
$$

The tension-test result finally becomes

$$
\left\langle t_{11}\right\rangle=2\left[1+\frac{3-\kappa}{4} G(h)\right] e_{11} .
$$

If the film is actually a plate, i.e., $h \rightarrow \infty$, the elasticity result is recovered, i.e.,

$$
\left\langle t_{11}\right\rangle=\frac{7-\kappa}{2} e_{11}=2(1+\nu) e_{11} \quad \text { (Elasticity), }
$$

where $2(1+\nu)$ is to be identified with Young's modulus, as $\operatorname{Dim} .\left\langle t_{11}\right\rangle=\mu\left\langle t_{11}\right\rangle$. The cohesive elasticity solution is

$$
\left\langle t_{11}\right\rangle=\left\{2(1+\nu)+\frac{3-\kappa}{2}[G(h)-1]\right\} e_{11} .
$$

It follows from (9.40) that the apparent modulus for a film is greater than Young's modulus and, in fact, is size dependent. It is widely known that the apparent strengths of some materials are effected by strain gradients. Using a couple-stress theory, which employs only a portion of the strain gradients, Mindlin [15] was able to show a reduction in stress-concentration factor around a small hole. The above result is consistent with the general belief that increasing strain gradients appear to make some materials stronger. Finally, the analysis of this section has essentially established the groundwork for a new plane-stress theory suitable for films.

10. Concluding remarks. The inclusion of higher-order gradients of displacements in a continuum theory leads to boundary-layer phenomena that are absent in all grade1 theories of which the ordinary theory of elasticity is a special case. This character may be exploited to facilitate the introduction of nontrivial self-equilibrating states, 
so that surface phenomena of solids may be defined by special displacement fields. If material isotropy is presumed, a theory must include at least the first three gradients of the displacements before a material constant can be introduced to capture the desired surface phenomena (Mindlin [17]). This is the grade-3 theory of Mindlin and the constant that gives rise to all the surface phenomena was termed the modulus of cohesion by him. It is for this reason that we have coined the name cohesive elasticity.

The most striking approach in Mindlin's formulation is the inclusion of the linear term $\varepsilon^{2} \beta_{0} E_{i i j j}$ in the strain energy-density $W$ of (3.17). It gives rise to a constant triple stress and leads to the concise energy expression $U^{*}$ of (3.27). This practice, however, is a matter of convenience in the sense that one could have left the term out of $W$ but introduced the constant triple stress as an initial or residual state. In fact, one could have interpreted the triple stress as a special body force in the sense of (7.28). The important point is that, however we interpret it, it is possible to introduce a new constant to generate a self-equilibrating state.

It is, of course, completely legitimate to include the linear term $E_{i i}$ in the elasticity version of strain energy-density. Such a term may also be interpreted as an initial or residual stress. The stress, however, must be completely relieved in the presence of a free boundary, as elasticity equations do not exhibit boundary-layer behavior and, as a result, the exercise leads to no useful results. It is obviously for this reason that a grain with an eigenstrain must interact with a nontrivial surrounding (Mura [21], Eshelby [8]). A nontrivial displacement field is associated with a free grain according to cohesive elasticity. We have analytically defined the meaning of grain boundary energy in Sec. 7. Unlike ordinary elasticity, cohesive elasticity requires the implementation of corner (boundary) conditions in case the boundary is not a smooth surface. In this connection, it is instructive to recall the presence of a corner force in a plate theory (a grade-2 theory). A notch- or crack-tip and a corner on a grain are among many meaningful problems to be analyzed. Even the meaning of a concentrated force may be reexamined. We have already found that the displacement under a point load is finite.

A grade- 2 theory contains couple stress and double stress. It is mathematically more tractable than a grade- 3 theory and also exhibits boundary-layer behavior. However, since it is not possible to include a term linear in $E_{i j k}$ in $W$, it is not possible to introduce a material constant to induce a self-equilibrating state for defining, say, surface free energy. On the other hand, one could introduce an initial or residual double stress, instead of triple stress, to induce a self-equilibrating system. The constant double stress is not a material constant any longer, but is still associated with a special chunk of a material. This possibility may turn out to be a simpler way of generating the desired surface phenomena.

Higher gradient theories were the popular topics of research of the sixties. Of the many known published results, Mindlin's work consistently placed the most emphasis on relating to real microscopic phenomena of solids. If the goal of today's micromechanics initiative is actually aiming at establishing physically relevant microcontinuum mechanics field theories, then Mindlin's works are indeed of a pioneering nature and should be carefully reexamined. 
Every time the word continuum is mentioned, the term constitutive relation follows. One of the commonly asked questions during the sixties was how to determine the so many constants involved in a grade- $n$ theory. It is our belief now that the negative implication of the question was a result of our preoccupation with the term constitutive relation which, in one way or another, is perceived as a response to a uniform sample. The existence of a uniform sample, say, the tension specimen, is a consequence of the assumption that the behavior of a solid is completely characterized by $E_{i j}$. While the concept of Cauchy stress and stress vector is not dependent on the former presumption, they are actually consistent in that the components of the surface traction vector would turn out to be just the appropriate components of the stress tensor if the latter were defined in terms of a strain energy-density via the stationary potential energy approach of Sec. 2. It is clear from Sec. 2 that once higher gradients are included the exact meaning of a Cauchy stress becomes vague and the direct connection between stress and stress vector $\mathbf{t}^{0}$ is gone. In general, there is no uniform sample to speak of anymore. Many new nontrivial problems of a more fundamental nature must first be solved before new test specimens can be devised. The surface free energy, unidirectional test solution, and point load solution (not included in this paper) are but a few of the anticipated test configurations.

No continuum field theories can be one hundred percent physical. For example, it could be argued that Young's modulus should be derived from more fundamental physical constants, but the "correct" Young's modulus is always measured from a tension specimen, which was designed in accordance with the mathematical solution that $\sigma=P / A$ and $\varepsilon=\Delta / L$. It is our belief that many, many more $P / A$ formulas must and will be discovered in the future. Finally, in connection with failure, the all important Eshelby tensor must be modified. The tensor itself could be straightforwardly defined by casting the spatial derivatives of $W$ into a divergence free form, but the associated conservation laws remain to be examined. It is even possible to couple the current grade- 3 theory with a higher-order diffusion equation, the Cahn-Hilliard equation (Cahn and Hilliard [2], Gurtin [13]), so that the modulus of cohesion will depend on concentration and one of the diffusion coefficients will depend on the triple stress. It is perhaps time again to reexamine the role of higher gradients in our pursuit for the understanding of failure of solids.

\section{REFERENCES}

[1] B. Budiansky and J. R. Rice, Conseriation laws and energy-release rates, J. Appl. Mech. 40, 201203 (1973)

[2] J. W. Cahn and J. E. Hilliard, Free energy of a nonuniform system I. Interfacial free energy, J. Chem. Phys. 28, 258-267 (1958)

[3] Julian D. Cole, Perturbation Methods in Applied Mathematics, Blaisdell, Waltham, Mass., 1968

[4] J. M. Doyle, Singular solutions in elasticity, Acta Mech. 4, 27-33 (1966)

[5] J. E. Dunn and J. Serrin, On the thermodynamics of interstitial working, Arch. Rational Mech. Anal. 88, 95-133 (1985)

[6] J. D. Eshelby, The force on an elastic singularity, Philos. Trans. Roy. Soc. London Ser. A 244, 87-112(1951)

[7] J. D. Eshelby, The continuum theory of lattice defects, Solid State Physics 3, (F. Seitz and D. Turnball, eds.), Academic Press, New York, 1956, pp. 79-144 
[8] J. D. Eshelby, The determination of the elastic field of an ellipsoidal inclusion and related problems, Proc. Roy. Soc. London, Ser. A 241, 376-396 (1957)

[9] J. D. Eshelby, Energy relations and the energy-momentum tensor in continuum mechanics, Inelastic Behavior of Solids, (M. F. Kannineu, W. F. Adler, A. R. Rosenfield, and R. I. Jaffee, eds.), McGraw-Hill, New York, 1970, pp. 77-114

[10] D. C. Gazis and R. F. Wallis, Surface tension and surface modes in semi-infinite lattices, Surface Science, vol. 3, 1964, pp. 19-32

[11] L. H. Germer, A. V. MacRae, and C. D. Hartman, (110) Nickel Surface, J. Appl. Phys. 32, 2432$2439(1961)$

[12] A. E. Green and R. S. Rivlin, Simple force and stress multipoles, Arch. Rational Mech. Anal. 16, 325-353 (1964)

[13] M. E. Gurtin, On a nonequilibrium thermodynamics of capillarity and phase, Quart. Appl. Math. 47, 129-145 (1989)

[14] J. K. Knowles and E. Sternberg, On a class of conservation laws in linearized and finite elastostatics, Arch. Rational Mech. Anal. 44, 187-211 (1972)

[15] R. D. Mindlin, Influence of couple-stresses on stress concentrations, Exp. Mech. 3, 1-7 (1963)

[16] R. D. Mindlin, Micro-structure in linear elasticity, Arch. Rational Mech. Anal. 16, 51-78 (1964)

[17] R. D. Mindlin, Second gradient of strain and surface-tension in linear elasticity, Internat. J. Solids Structures 1, 417-438 (1965)

[18] R. D. Mindlin, On the equations of elastic materials with micro-structure, Internat. J. Solids Structures 1, 73-78 (1965)

[19] R. D. Mindlin, Polarization gradient in elastic dielectrics, Internat. J. Solids Structures 4, 637-642 (1968)

[20] R. D. Mindlin and N. N. Eshel, On first strain-gradient theories in linear elasticity, Internat. J. Solids Structures 4, 109-124 (1968)

[21] T. Mura, Micromechanics of Defects in Solids, Martinus Nighoff Publishers, The Hague, The Netherlands, 1982

[22] L. E. Murr, Interfacial Phenomena in Metals and Alloys, Addison-Wesley, Reading, Mass., 1975

[23] G. C. Sih and H. Liebowitz, On the Griffith energy criterion for brittle fracture, Internat. J. Solids Structures 3, 1-22 (1967)

[24] R. A. Toupin, Elastic materials with couple-stress, Arch. Rational Mech. Anal. 11, 386-414 (1962)

[25] R. A. Toupin and D. C. Gazis, Surface effects and initial stress in continuum and lattice models of elastic crystals, Proceedings of the International Conference on Lattice Dynamics, Copenhagen, (R. F. Wallis, ed.), Pergamon Press, New York-Oxford, 1964, pp. 597-602

[26] M. Van Dyke, Perturbation Methods in Fluid Mechanics, Parabolic Press, Stanford, California, 1975 